\title{
Fibre-direction strain measurement in a composite ply under pure bending using Digital Volume Correlation and Micro-focus Computed Tomography
}

\author{
E. Schöberl ${ }^{1}$, M. N. Mavrogordato ${ }^{1}$, I. Sinclair ${ }^{1}$, S. M. Spearing ${ }^{1}$ \\ ${ }^{1}$ Engineering Materials, Faculty of Engineering and Physical Sciences, University of Southampton, \\ Southampton SO17 1BJ, UK
}

Keywords: Carbon-Fibre Reinforced Polymers (CFRPs), Microstructures, Micromechanics, Microfocus Computed Tomography $(\mu \mathrm{CT}$ ), Digital Volume Correlation (DVC), Strain mapping

\section{Abstract}

This paper presents an experimental demonstration and validation of high-resolution threedimensional experimental strain measurement using Digital Volume Correlation (DVC) on CarbonFibre Reinforced Polymers (CFRPs), via through-thickness strain analysis under a state of pure bending. To permit the application of DVC to displacements and/or strain measurements parallel to the fibre direction in well-aligned unidirectional (UD) materials at high volume fractions, a methodology was developed for the insertion of sparse populations of $400 \mathrm{~nm} \mathrm{BaTiO}_{3}$ particles within the matrix to act as displacement trackers (i.e. fiducial markers). For this novel material system, measurement sensitivity and noise are considered, along with the spatial filtering intrinsic to established DVC data processing. In conjunction with Micro-focus Computed Tomography $(\mu \mathrm{CT})$, the technique was applied to a simple standard specimen subjected to a four-point flexural test, which resulted in a linear strain distribution through the beam thickness. The high-resolution, fibre-level strain distributions (imaged at a voxel resolution of $\sim 0.64 \mu \mathrm{m}$ ) were compared against the classical beam theory (Euler-Bernoulli) in incrementally decreasing averaging schemes and different sub-set sizes.

Different sampling and averaging strategies are reported, showing that DVC outputs can be obtained that are in very good agreement with the analytical solution. A practical lower limit for the spatial resolution of strain is discerned for the present materials and methods. This study demonstrates the effectiveness of DVC in measuring local strains parallel to the fibre direction, with corresponding potential for calibration and validation of micromechanical models predicting various fibre-dominated damage mechanisms.

\section{Introduction}

Climate change continues to be a global challenge. In December 2015, the Paris agreement [1] established goals to limit temperature increase to less than $2^{\circ} \mathrm{C}$, with ambitions to less than $1.5^{\circ} \mathrm{C}$. Owing to high specific mechanical properties and the potential to engineer structural properties to precise applications and loading conditions, Carbon-Fibre Reinforced Polymers (CFRPs) have been identified as a pivotal technology for lightweighting across the transport sector [2], where weight reduction remains the fundamental approach to reducing greenhouse gas emissions (e.g. $\left.\mathrm{CO}_{2}, \mathrm{NO}_{\mathrm{x}}\right)$.

Due to the multiphase composition, anisotropy and heterogeneity of composite laminates, failure prediction remains difficult, even under the simplest loading conditions. As such, a fundamental understanding of the fibre fracture process is important for a complete interpretation of composite tensile failure, alongside the various other forms of damage that develop in these materials under load.

Characterizing volumetric material deformation and damage, is however, notoriously challenging, due to the opacity of the materials, the three-dimensional (3D) multi-scale nature of damage, the 
coupling of multiple micromechanisms and the difficulty of distinguishing damage caused by load from cutting and polishing artefacts associated with traditional metallographic approaches. A significant step forward has been achieved in recent years by the use of X-ray Computed Tomography (CT) combined with in situ loading to identify detailed sequences of damage accumulation down to fibre-level, in 3D, within the bulk of representative materials under load [3], [4], [5], [6], [7]. As a natural evolution, the coupling of CT with Digital Volume Correlation (DVC) was identified.

An extension of the white-light illumination, two-dimensional (2D) Digital Image Correlation (DIC) technique [8], DVC was developed for volumetric measurements, and initially demonstrated as a means to estimate the effective continuum strain tensor in trabecular bone subjected to axial compression [9]. The general objective of the DVC is to track a pattern of the reference volume in the deformed volume image, and to determine its associated shift vector. This may be achieved by employing a correlation criterion (function) to search for a digital pattern within a time-series volumetric data set. In case of volume images, the pattern is given by an array of voxel grey-level intensities, i.e. a contrast pattern [9], [10]. As a result, DVC is a tool capable of quantifying the internal microstructural response of the material between different load states, by extracting essential failure parameters (e.g. local displacements and strains) [11].

In the field of composite mechanics the application of DVC is less extensive, but previous work includes it's use as a strain-based quantification of the deformation fields to investigate: strain concentrations and strain transfer lengths associated with the fracture of continuous-fibres [12], [13], free-edge effects [14], Mode I delamination-induced damage in particle-toughened interlayers [15] and transverse shear effects [16]. DVC therefore appears to be a powerful tool for measuring volumetric displacements and computing strains, but for X-ray CT, the noise and sensitivity of the measurements rely heavily on a series of factors. These include imaging hardware (beam stability, flux, energy, exposure, propagation distance, voxel size), the nature of the material type under investigation (sample shape, size, phase density distribution, trackable features) and imaging results (contrast-to-noise ratio, spatial resolution, artefacts), all of which influence the quality of the image volumes obtained [17], [18].

Consequently, an assessment of the reliability and accuracy of the measurements is required through validation experiments, where the displacement and/or strain field is known a priori [17]. The approach commonly involves two zero-strain pair analyses: a static repeat scan and a rigid body displacement assessment [12], [13], [15], [18], [19], [20].

In the context of CFRP tensile failure [12], [13], and progressing from the conventional noise tests, the authors propose a more comprehensive validation study, specifically focused on an unidirectional (UD) material, through the application of a four-point flexural test to a simple standard specimen with a well-defined strain distribution. The advantage of this approach is that the strain gradient is linear through beam thickness, related directly to the curvature (i.e. the reciprocal of the radius of curvature [21]) which is easily measured and does not need measurements of load or material elastic properties. Furthermore, this approach allows the exploration of the effects of averaging and the selection of subset size on the accuracy of the gradient of strain values.

The highly anisotropic and somewhat regular/self-similar microstructures found in conventional unidirectional CFRPs at high volume fractions are intrinsically challenging for DVC, particularly along the fibre direction. The cylindrical structure and smooth surfaces of the filaments lack a well-defined, trackable, contrast pattern along the fibre direction, leading to poor correlation along the fibres, and correspondingly inaccurate displacement estimates in this direction within a given ply [15]. Following a similar approach to that taken by Brault et al. [16] to generate individual features unique to a particular sub-set, the authors have explored the insertion of sparse populations of significantly smaller particles ( $400 \mathrm{~nm}$ as opposed to $150 \mu \mathrm{m}$ ) within the matrix to act as displacement trackers - i.e. fiducial markers. While the particles may have an impact on the mechanical performance of the material, it can be 
considered negligible especially in the context of this paper involving strain mapping under elasticbending conditions. High-resolution, fibre-level strain distributions are specifically addressed (imaged at a voxel resolution of $\sim 0.64 \mu \mathrm{m}$ ), as opposed to the ply-level studies in [16] (which used a voxel resolution of $52 \mu \mathrm{m}$ ). This paper reports on the development of the fiducial-adapted CFRP, corresponding Micro-focus CT $(\mu \mathrm{CT})$ four-point flexural testing and DVC processing: micromechanical strain mapping results within the fibrebed, to evidence the utility of the technique at very much higher resolutions than reported in the literature.

\section{Materials and methods}

\subsection{Material manufacturing}

Cross-ply laminates, with a [90/0 $]_{\mathrm{s}}$ layup, height $(h)$ of $\sim 1 \mathrm{~mm}$ and a nominal fibre volume fraction $\left(V_{f}\right)$ of $\sim 55 \%$ were manufactured by drum winding at KU Leuven, Belgium. A polyacrylonitrile-based (PAN) 12K TORAYCA T700SC-50C (Toray Industries Inc., Tokyo, Japan [22]) non-twisted tow was used, with a $7 \mu \mathrm{m}$ nominal fibre diameter.

To create microstructural fiducial patterns for the application of DVC, the resin was filled with commercially available tetragonal $\mathrm{BaTiO}_{3}$ particles, nominal mean size of $400 \mathrm{~nm}$ and approximately spherical shape (US Research Nanomaterials Inc., Houston, TX, USA [23]). This property combination was selected following an extensive $\mu \mathrm{CT}$-based qualitative assessment on a range of possible fiducial compositions ( $\mathrm{Al}, \mathrm{Al}_{2} \mathrm{O}_{3}, \mathrm{SiC}, \mathrm{SiO}_{2}, \mathrm{MgO}, \mathrm{TiC}, \mathrm{TiO}_{2}, \mathrm{BaCO}_{3}, \mathrm{BaTiO}_{3}, \mathrm{Cu}$ and $\mathrm{Bi}_{2} \mathrm{O}_{3}$ ), mean particle sizes ( $300 \mathrm{~nm}$ to $800 \mathrm{~nm}$ ) and concentrations ( $0.25 \mathrm{wt}$. \% to $15 \mathrm{wt}$. \% of the resin). From this test matrix, $\mathrm{BaTiO}_{3}$ particles were found to offer the most favourable compromise between contrast in CT images (high attenuation coefficient) and the ability to obtain a homogeneous distribution in 3D space with sufficient particle compactness for local DVC analyses. The particles were dispersed in a SiPreg KTA 313/SR 8500 dual-component epoxy (Sicomin, Châteauneuf-les-Martigues, France [24]) using a combination of high-shear mixing and heated ultra-sonication (U100H bath, Ultrawave Ltd., Cardiff, UK [25]). No specific treatment was applied to the particle surfaces. To remove entrapped air, the thermosetting mixture was degassed for 10 minutes at ambient temperature. The amount of resin used was $150 \mathrm{~g}$, with $31.5 \mathrm{~g}$ of hardener, following the manufacturer's specified mixing ratio of 100/21 (by weight). The $\mathrm{BaTiO}_{3}$ fraction was set at $7.5 \mathrm{wt}$. \% of the resin, equivalent to $\sim 1.44$ vol. \%. A nominal $25 \%$ tow overlap was targeted during the winding process. A maximum spool tension of $0.12 \mathrm{lb}$ (54.43 g) was applied. To control the volume fraction of the matrix, following impregnation, the tow was passed through a flat die with an orifice of $0.2 \mathrm{~mm} \times 9 \mathrm{~mm}$. The temperature of the tow spreader and final guide roller were set to $50^{\circ} \mathrm{C}$. The fibre sizing was left intact. A schematic of the manufacturing setup is shown in Fig. 1. The drum winding process resulted in uniaxial prepreg tape, $\sim 0.25 \mathrm{~mm} \mathrm{x} \sim 350$ $\mathrm{mm} \mathrm{x} \sim 1900 \mathrm{~mm}$, which was cut and laid up to produce the desired [90/0 $]_{\mathrm{s}}$ cross-ply layup. The prepreg stack was cured in an autoclave for 280 minutes at $0.5 \mathrm{MPa}(5$ bar $)$ and $120^{\circ} \mathrm{C}$. To minimize void content in the cured material, a vacuum of $\sim 0.07 \mathrm{MPa}(\sim 0.7$ bar) was maintained throughout the autoclave process. 


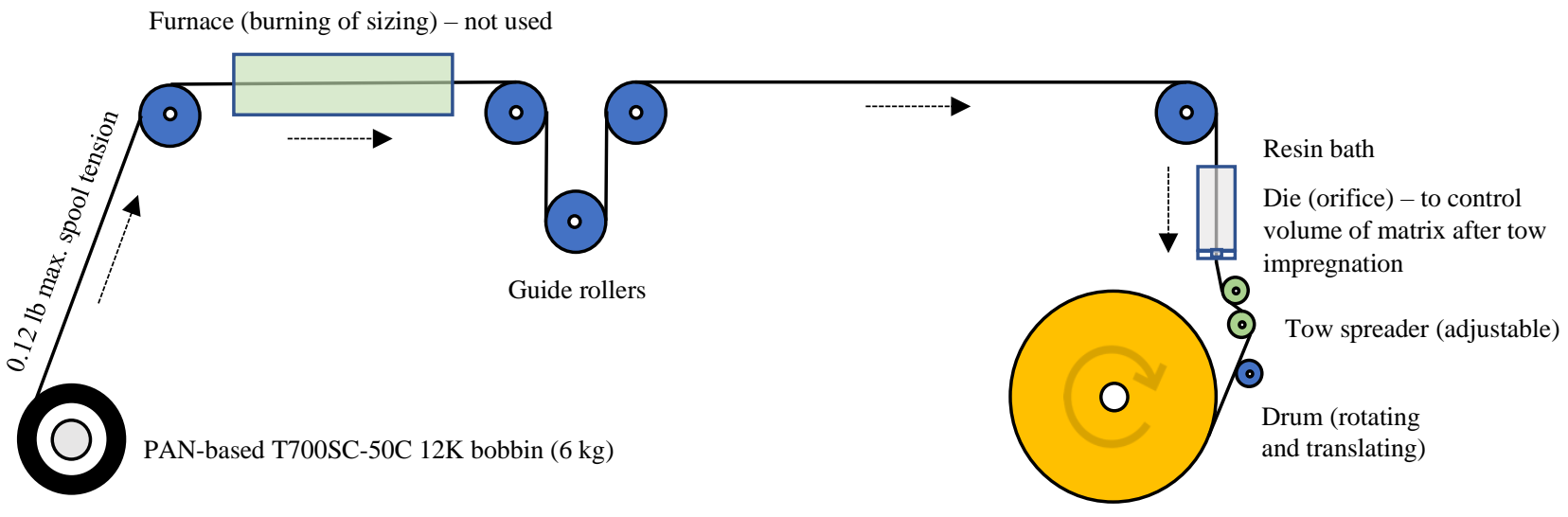

Figure 1 - Schematic illustration of the prepreg manufacturing (drum winding) process.

\subsection{Specimen geometry and loading}

Specimen preparation was carried out in accordance with the D 7264/D 7264M - 07 test method [26]. A standardized support-span $(L)$ to thickness $(t)$ ratio of 20:1 was used. 'Matchsticks' $(\sim 1 \mathrm{~mm} \mathrm{x}$ $\sim 41.5 \mathrm{~mm} \mathrm{x} \sim 1 \mathrm{~mm}$ ) were prepared via water-jet cutting from the manufactured plates described in Section 2.1. The specimens were cut with additional length to ensure good alignment with the loading and support span respectively. The specimen geometry, region of interest (ROI) and direction of loading relative to the composite layup are shown in Fig. 2.

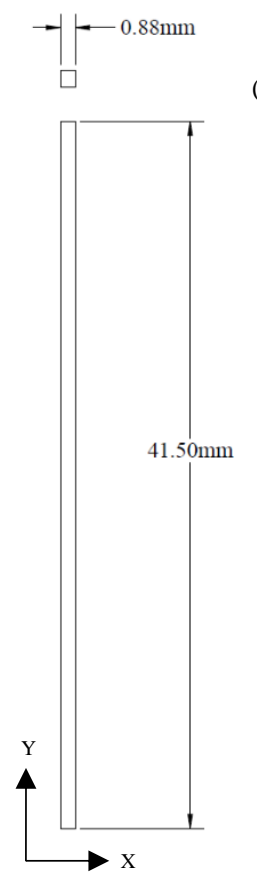

(a)

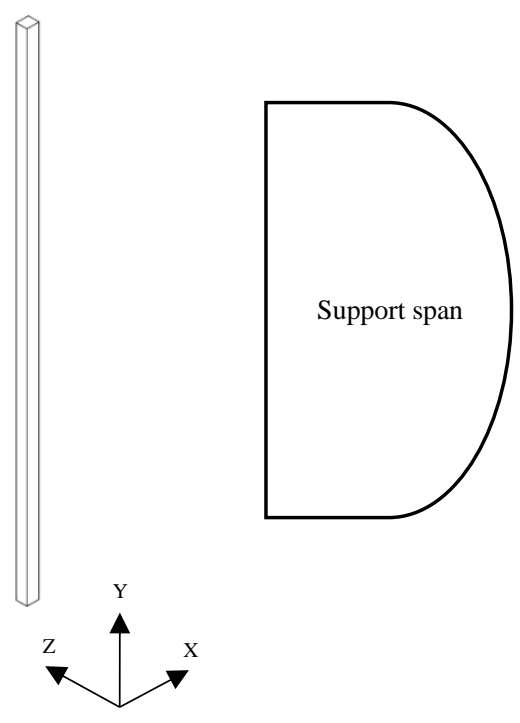

(b)

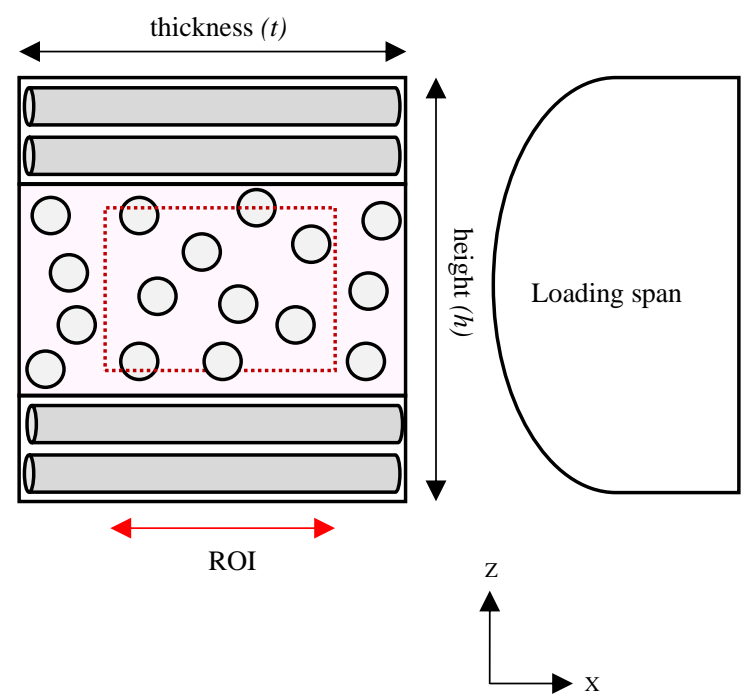

Figure 2 - (a) Specimen geometry (thickness as measured via CT), and (b) direction of loading relative to the composite layup (orthogonal to the $0^{\circ}$ plies $-X Z$-plane shown). The ROI shown schematically corresponds to that of the cropped CT volume for DVC analysis.

The specimen was enclosed in a $58 \mathrm{~mm}$ long, $7 \mathrm{~mm}$ ID and $10 \mathrm{~mm}$ OD Poly(Methyl Methacrylate) - PMMA reaction tube, illustrated in Fig. 3 (a). A polymer-based material was selected for the construction of the tube, to minimize X-ray absorption. Holes were machined and threaded to 
accommodate M3 grub-screws to act as the support $(20 \mathrm{~mm})$ and loading span $(10 \mathrm{~mm})$ respectively. Opposite the loading span, two cavities were designed to allow manipulation of the specimen once the tube was capped with the 3D printed Acrylonitrile Butadiene Styrene (ABS), non-load bearing wedges - shown in Fig. 3 (b). Guide-grooves $(1.10 \mathrm{~mm} \times 2.0 \mathrm{~mm})$ were designed in the top and bottom wedges, aligned with the loading screws. A tolerance of $0.25 \mathrm{~mm}$ was provided around the specimen ( $\mathrm{Y}$ - and Zdirection) to ensure that it did not come into contact with the wedge surfaces, thereby influencing the bending deformation. The wedges were designed with interlocking pins to ensure both good alignment and no movement relative to the tube. The assembly, as shown in Fig. 3 (c) was mounted onto the sample manipulator stage via a stand-off that screwed directly into the bottom wedge.

(a)

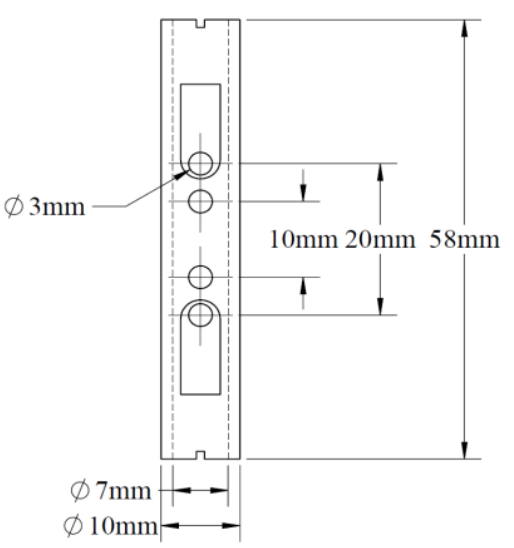

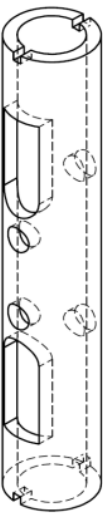

(b)

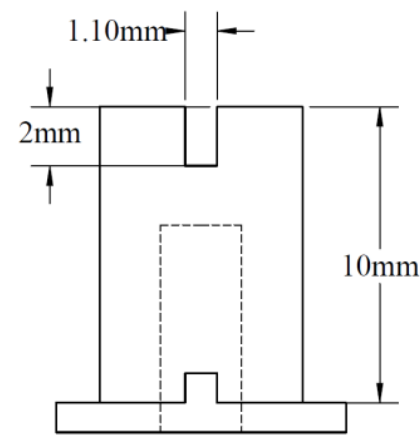

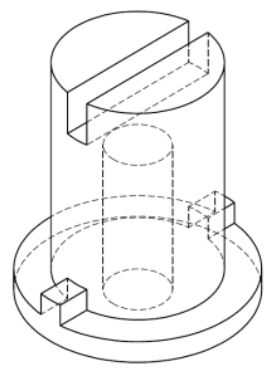

(c)

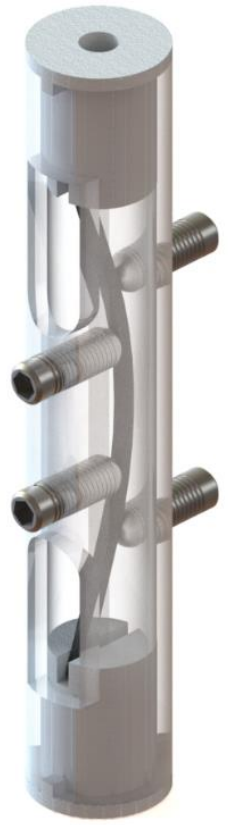

Figure 3 - (a) Reaction tube with relevant dimensions, (b) wedges designed to maintain optimum alignment of the specimen, (c) complete assembly - showing both a bent and unbent geometry to illustrate the operating principle.

The bending of the specimen was applied ex situ, by manually rotating both the grub screws simultaneously via a pair of Allen keys. Rotation of the screws was carried out in increments of $1 / 8^{\text {th }}$ of 
a turn until the desired curvature was reached. A relatively small deformation (large radius of curvature) was intended in order to ensure that (a) the classical beam theory (Euler-Bernoulli) is applicable [21], and (b) to prevent microstructural damage occurring within the two $0^{\circ}$ plies (e.g. fibre fractures, intra/interlaminar matrix cracks and delaminations) as such damage would significantly alter the local strain distribution [12], [13], [15]. Conversely, the radius of curvature attained was selected to be small enough, for the maximum strain within the ROI to exceed the anticipated experimental noise. A single load step (sequence) was performed.

\subsection{Computed Tomography}

Micro-focus CT measurements were carried out at the $\mu$-VIS Imaging Centre at the University of Southampton, UK. Scans were conducted in a Xradia 510 Versa (Carl Zeiss AG, Oberkochen, Germany [27]) polychromatic system, equipped with a tungsten transmission target. A 2048 x 2048-pixel detector was used, with a chip size of $13.5 \mu \mathrm{m}$. To achieve the desired voxel resolution levels, a two-stage magnification approach was implemented (RaaD [27]), combining the geometric magnification of the $\mathrm{X}$-Ray imaging with a set of barrel-mounted lenses. Scans on the specimen were conducted at different resolutions, depending on the designation: (a) low-resolution - with greater field of view (FOV) for the sole purpose of determining the radius of curvature, and (b) high-resolution - used in order to determine fibre-level strain gradients through the application of DVC. The low-resolution scans were acquired through an objective with $4 \mathrm{x}$ magnification, yielding a voxel size of $\sim 4.02 \mu \mathrm{m}$ for the bent and unbent specimen respectively. The high-resolution scans were conducted at 20x magnification, yielding a voxel size of $\sim 0.64 \mu \mathrm{m}$ for the bending configuration and noise assessment scans (static repeat and rigid body displacement) respectively. A $1 \mathrm{~s}$ exposure was used for the low-magnification scans. The number of projections per scan was set to 1601 , giving a duration of $\sim 0.44 \mathrm{hrs}$ per tomograph. For the highmagnification scan, the exposure time was increased to $30 \mathrm{~s}$, resulting in a scan time of $\sim 13.34 \mathrm{hrs}$. The beam energy was set to $80 \mathrm{kVp}$ and the current to $87 \mu \mathrm{A}$ throughout. A 2x detector 'binning' was used, with acquisition taking place over a rotation of $\pm 180^{\circ}$. The FOV corresponds to $\sim 4 \mathrm{~mm} \mathrm{x} \sim 4 \mathrm{~mm} \mathrm{x}$ $\sim 4 \mathrm{~mm}$ and $\sim 0.58 \mathrm{~mm} \mathrm{x} \sim 0.62 \mathrm{~mm} \mathrm{x} \sim 0.64 \mathrm{~mm}$ for the low- and high-resolution scans respectively. A source-to-object and object-to-detector distance of $\sim 17 \mathrm{~mm}$ and $\sim 18 \mathrm{~mm}$ respectively was used. The data was reconstructed using conventional absorption-based Filtered Back Projection (FBP). Fig. 4 illustrates the complete experimental setup.

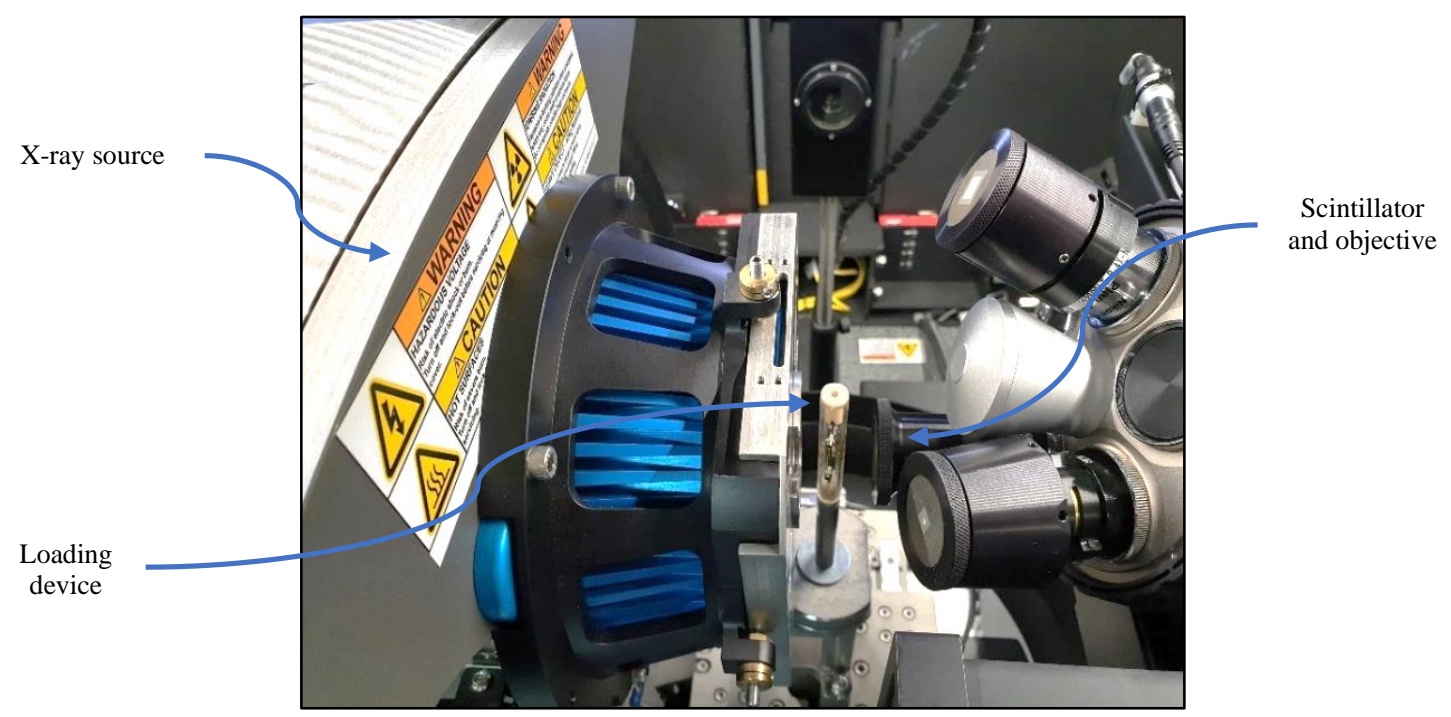

Figure 4 - Experimental setup illustrating the loading device in acquisition position. 


\subsection{Digital Volume Correlation}

Digital Volume Correlation was performed using the commercial DaVis v10 software with StrainMaster DVC package (LaVision GmbH, Göttingen, Germany [28]). StrainMaster employs a correlation criterion operating on the grey-level intensity values of 3D images [29]. In essence, the measurement volume is divided into smaller sub-sets, and the contrast pattern within each sub-set is then tracked from the reference to the deformed state independently (local approach [30]) as a discrete function of the grey-levels [29]. A cross-correlation function is employed to measure the conservation of the grey-levels between the original and displaced sub-set, with the position determined for which the correlation is closest to unity [15], [18], [29]. Analogous to DIC, the correlation coefficient can be used to evaluate the similitude between the sub-sets [18]. A coefficient of 1.0 implies that the sub-sets are completely related, whereas a value of 0 indicates that they are completely unrelated [18].

The workflow of the correlation process is illustrated schematically in Fig. 5. The associated shift (3D displacement) is given by the vector connecting the sub-set centroids between the deformation states [28]. Finally, 3D strain field estimation may be carried out through a centred finite difference to determine the numerical derivatives of the vector field [18], [20], [29], [31].

Recognition of matching sub-sets between the deformation states was performed within the DVC software via a multi-step, multi-pass strategy, whereby a global pre-shift was computed through a Fast Fourier Transform (FFT) approach, followed by Direct Correlation (DC). The approach maximised the efficiency of using the DVC algorithm by exploiting the $\mathrm{BaTiO}_{3}$ particles as fiducial markers along the fibre direction, as the search for the corresponding sub-set in the deformed state was confined to a shift equal to the search radius (Fig. 6). Furthermore, the approach allowed for the coarse displacements captured by larger sub-sets to be used as a predictor vector field for the subsequent and refined iterations, involving smaller sub-sets.

Both implementations use an equivalent of a piece-wise linear shape function for the referencedeformed mapping, and a normalized form of the cross-correlation function to quantify the conservation of the grey-levels between the images [18], [28], [29], [32]. A trilinear interpolation is used in 'FFT', and a third-order spline interpolation in 'DC', to compute the greyscale intensities of each displayed voxel at non-integer positions (i.e. sub-voxel measurements) [32]. 'DC' also permits the use of a weighted window within sub-sets, an option which was implemented during the final iteration step of correlation (dual-pass). This results in a potentially improved representation of the local displacement field, as the voxels in the vicinity of the sub-set centroid carry more weight than voxels located at the edge of the sub-set. While the weighted window extends over two times the sub-set size, the implementation ensures that the effective spatial resolution is similar to that of a conventional sub-set, for which an arithmetic average is implemented [28].

As isotropic sub-sets were used throughout all present analyses, a specific notation is introduced, whereby the specified size of a sub-set is equivalent to a sub-set with its characteristic length cubed (i.e. sub-set size of $N$ voxels is equivalent to a sub-set size with $N \times N \times N$ voxels or $N^{3}$ voxels). 

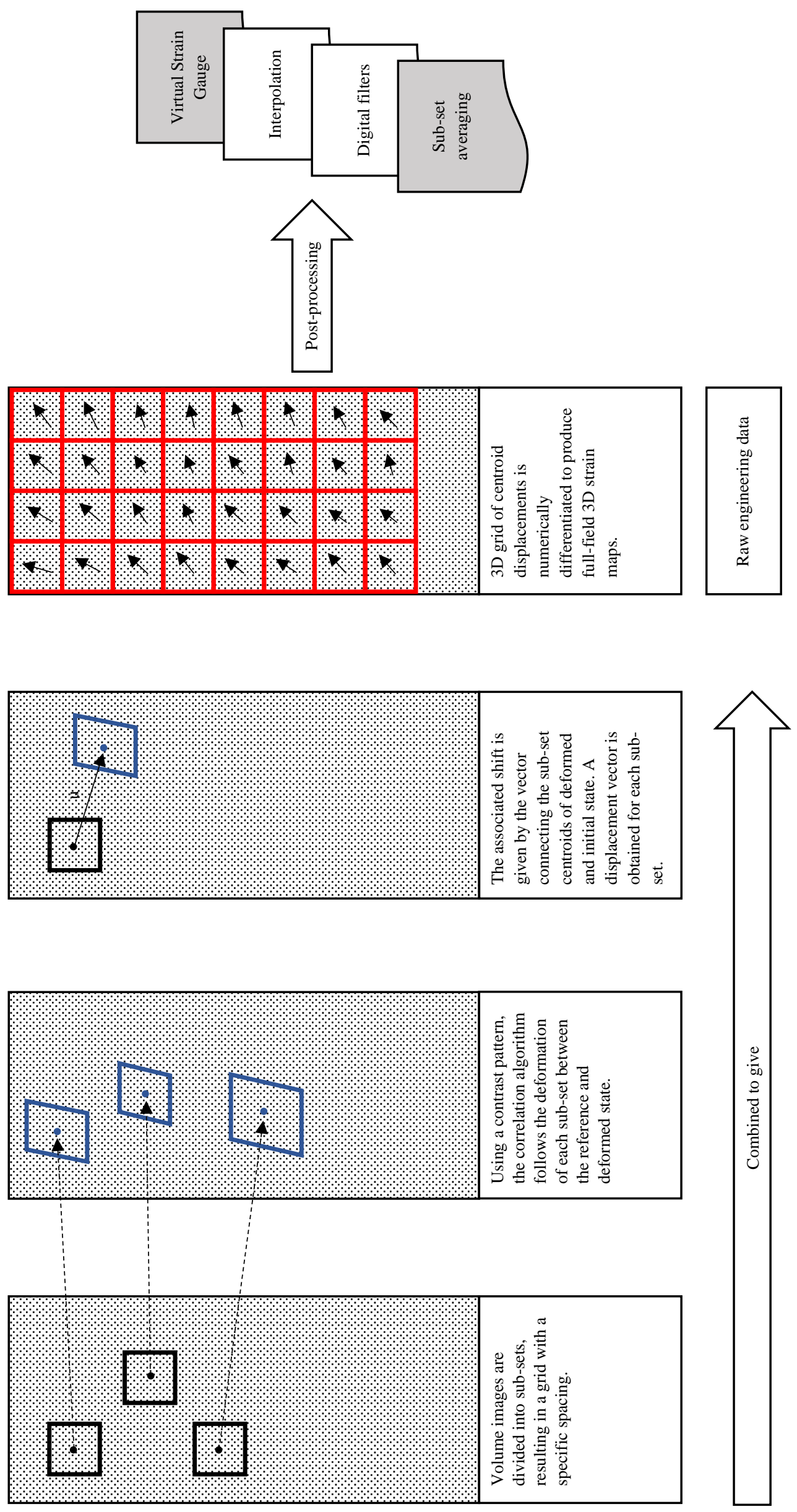


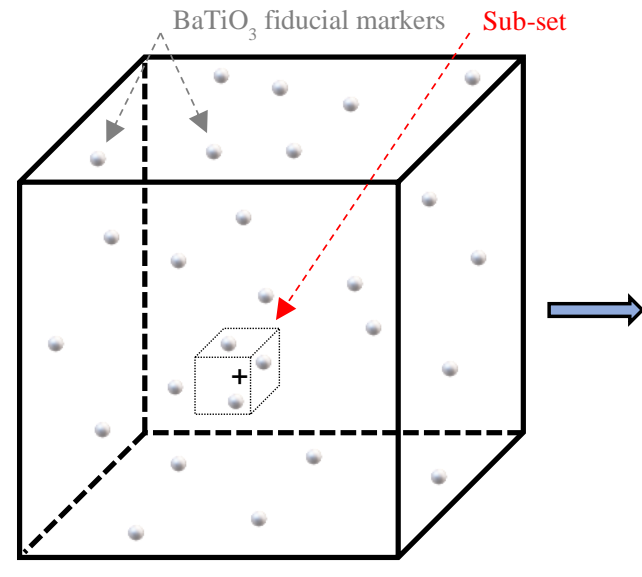

Undeformed volume image (A)

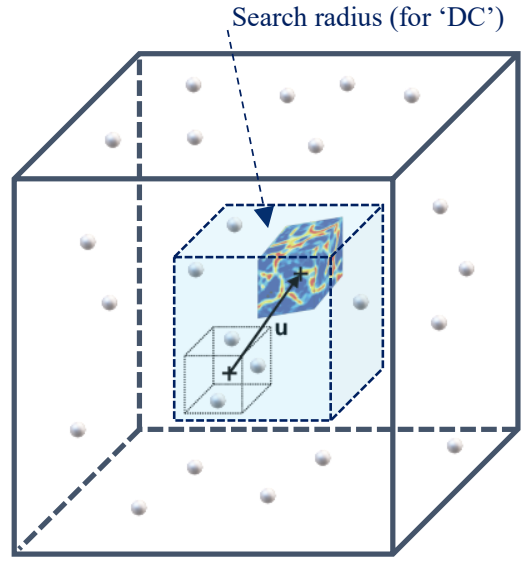

Deformed volume image $(B)$

Figure 6 - Schematic diagram of the DVC process: inclusion of fiducials and presence of a user-specific search region when using the 'DC' method. Figure not to scale. Adapted from Xu [11] with permission from Elsevier and with permission from Jiroušek et al. [33].

To moderate the computational cost, two further strategies were implemented. First, a 16-bit to 8bit integer conversion was performed. While the correlation results will always be imaging configuration dependent, Buljac et al. [34] have shown that histogram (grey-level) rescaling has negligible impact on the DVC error. Similarly, the bit-depth of volumes was also reduced in [15] to achieve faster processing times. Secondly, the search radius was incrementally reduced from 8 to 6 and ultimately 4 voxels between the deformed and undeformed state. As reported in [12] and [13], the latter value corresponds to a typical fibre-break opening (based on a $0.65 \mu \mathrm{m}$ voxel size) in a T700SC50C/epoxy-based cross-ply, implying that in the absence of fractured filaments and/or matrix cracks within the $0^{\circ}$ plies, the elastic response of the microstructure cannot exceed the aforementioned displacement level.

To increase sampling, the overlap between the neighbouring sub-sets was set to $50 \%$, as reported in [15], [18] and [20]. This is less than the $75 \%$ overlap reported in [12], [13], however it is desirable to balance the poorer signal-to-noise available using the present materials and methods - Section 3.1.2. The relevant settings used are detailed in Table 1.

\begin{tabular}{|c|c|c|c|c|c|c|c|c|}
\hline $\begin{array}{l}\text { Final sub-set size } \\
\text { (voxels) }\end{array}$ & $\begin{array}{l}\text { FFT pre-shift } \\
\text { (voxels) }\end{array}$ & $\begin{array}{c}\text { Step } 1 \\
\text { (voxels) }\end{array}$ & $\begin{array}{c}\text { Step } 2 \\
\text { (voxels) }\end{array}$ & $\begin{array}{c}\text { Step } 3 \\
\text { (voxels) }\end{array}$ & $\begin{array}{c}\text { No. of } \\
\text { passes } \\
\text { steps 1-3 }\end{array}$ & $\begin{array}{c}\text { Voxel } \\
\text { binning } \\
\text { step } 1\end{array}$ & $\begin{array}{c}\text { Voxel } \\
\text { binning } \\
\text { step } 2\end{array}$ & $\begin{array}{c}\text { Voxel } \\
\text { binning } \\
\text { step 3 }\end{array}$ \\
\hline 40 & 80 & 80 & 48 & 40 & \multirow{9}{*}{2} & \multirow{9}{*}{$4 \times 4 \times 4$} & \multirow{9}{*}{$2 \times 2 \times 2$} & \multirow{9}{*}{ NONE } \\
\hline 48 & 80 & 80 & 56 & 48 & & & & \\
\hline 60 & 96 & 96 & 64 & 60 & & & & \\
\hline 72 & 128 & 128 & 88 & 72 & & & & \\
\hline 80 & 128 & 128 & 96 & 80 & & & & \\
\hline 100 & 144 & 144 & 112 & 100 & & & & \\
\hline 120 & 176 & 176 & 136 & 120 & & & & \\
\hline 148 & 192 & 192 & 160 & 148 & & & & \\
\hline 156 & 192 & 192 & 160 & 148 & & & & \\
\hline
\end{tabular}

Table 1 - Summary of 'DC' settings used in the multi-step, multi-pass approach (50\% sub-set overlap).

Weighted windows used in the last iteration step (dual-pass). 
To assess the noise and sensitivity associated with the DVC technique, conventional stationary and rigid body displacement tests were performed (Section 3.1.1. and 3.1.2.). DVC was applied to cropped volumes from the $0^{\circ}$ plies, measuring $795 \times 885 \times 230$ voxels. As previously indicated in Fig. 2, the Yaxis indicates the fibre direction, the $\mathrm{X}$-axis is in-plane orthogonal to the fibre direction, while the Zaxis corresponds to the out-of-plane direction. The anisotropic volume represented the maximum available window to perform DVC, particularly limited in the Z-direction by the position of the FOV relative to the centre of the specimen and the height $(h)$ of $0^{\circ}$ plies. Volumes were carefully checked in advance against the presence of any significant $\mathrm{CT}$ artefacts and/or variations in sharpness within the visible region.

As summarized in Table 2, the number of DVC 'slices' (i.e. planes composed of volumetric subsets, one sub-set thick) is computed by dividing the height of the cropped volume (230 voxels) by the step size used in the correlation algorithm. Such an example is illustrated in Fig. 7, where a stack comprising of four DVC slices can be observed. Likewise, the number of in-plane data points per DVC slice can be computed by dividing the sub-set plane dimensions ( 795 x 885 voxels) by the step size Table 3.

Sub-sets positioned at the perimeter of the grid (XY-plane) as well as the planes of sub-sets located at the top ends of the stack (Z-direction) must be treated with special consideration (e.g. masked or truncated) if displacement vectors are computed, but not $100 \%$ satisfied from a voxel validity perspective - i.e. notional sub-sets that overlap with the edge, due to cropping between deformation states and/or large rigid body displacements, potentially causing the correlated data to be noisier [20] or incomplete.

\begin{tabular}{|c|c|c|c|}
\hline Sub-set size (voxels) & Step size (voxels) & $\begin{array}{c}\text { No. of DVC slices } \\
\text { (Z-direction) } *\end{array}$ & $\begin{array}{c}\text { No. of raw data slices per } \\
\text { DVC slice }\end{array}$ \\
\hline 80 & 40 & 6 & 40 \\
\hline 100 & 50 & 5 & 50 \\
\hline 120 & 60 & 4 & 60 \\
\hline 148 & 74 & 3 & 74 \\
\hline
\end{tabular}

Table 2 - Number of DVC slices (planes of sub-sets) for a given sub-set size with $50 \%$ overlap, and the corresponding number of raw data slices per DVC slice. *Nomenclature: DVC slice numbering starts with 0. 


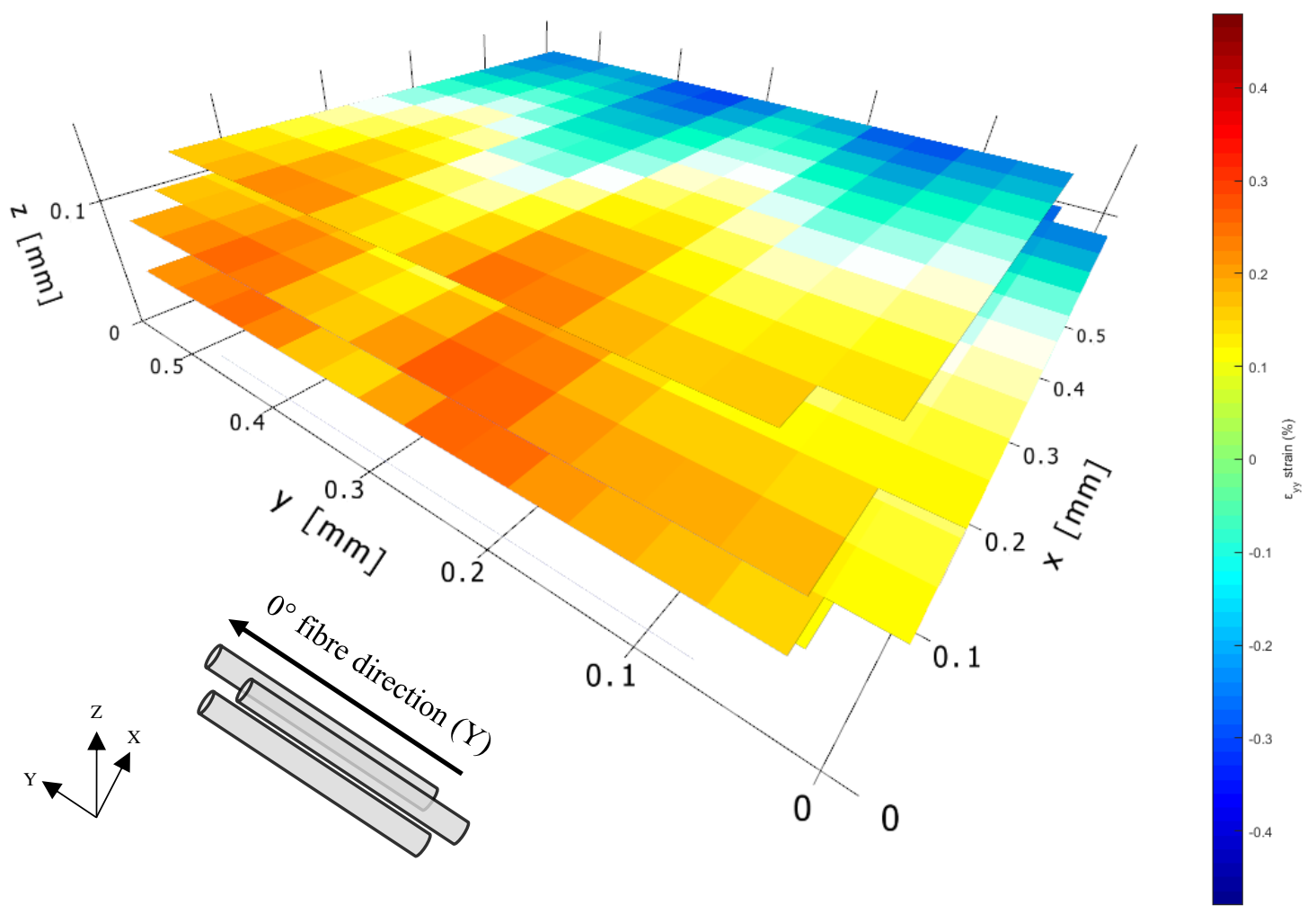

Figure 7 - Example stack comprised of 4 DVC slices in the $Z$-direction ( ' $Z=0$ ' to ' $Z=3$ '), applicable to a sub-set size of 120 voxels with $50 \%$ overlap. In turn, each DVC slice (i.e. plane of sub-sets, one volumetric sub-set thick) is representative for 60 raw CT data slices. Raw data volumes cropped from the $0^{\circ}$ plies: $795 \times 885 \times 230$ voxels. $Y$-axis indicates the fibre direction. $X$-axis is in-plane orthogonal to the fibre direction, while the Z-axis corresponds to the out-of-plane direction.

\begin{tabular}{|c|c|c|c|c|}
\hline Sub-set size (voxels) & Step size (voxels) & $\begin{array}{c}\text { No. of DVC slices } \\
\text { (Z-direction) }\end{array}$ & $\begin{array}{c}\text { No. of data points per } \\
\text { slice } \\
\text { (X-direction) }\end{array}$ & $\begin{array}{c}\text { No. of data points } \\
\text { per slice } \\
\text { (Y-direction) }\end{array}$ \\
\hline 80 & 40 & 6 & 20 & 22 \\
\hline 100 & 50 & 5 & 16 & 18 \\
\hline 120 & 60 & 4 & 13 & 15 \\
\hline 148 & 74 & 3 & 11 & 12 \\
\hline
\end{tabular}

Table 3 - Number of in-plane data points (X-and Y-direction) per DVC slice, applicable to a sub-set with $50 \%$ overlap.

A common post-processing aspect of strain computation via DVC (and related DIC algorithms) is the Virtual Strain Gauge (VSG). The VSG defines the local (effective) region of the image that is used for strain calculation at a specific location and is volumetrically analogous to the physical area that a metallic foil strain gauge covers [31]. Additionally, the VSG may also be considered to be a smoothing/denoising strategy as it represents a higher-order filtering stage for the computed strains. More details about the application of VSGs can be found in [31].

Several key variables affect the VSG, such as the characteristic length (size) of the: strain window $\left(L_{S W}\right)$, step $\left(L_{S T}\right)$ and sub-set $\left(L_{S S}\right)$. As the characteristic length $\left(L_{V S G}\right)$ of the VSG decreases, the noise typically increases, as the amount of global spatial filtering decreases. Similarly, as the size of the VSG 
decreases, the maximum strain amplitude along a line cut (extracted region of highest strain gradient) will generally increase [31]. In this paper, a VSG with an $L_{S W}$ of $3 \times 3 \times 3$ and $3 \times 3 \times 1$ neighbouring vectors was used for volumetric and planar strain computation respectively. The application of a VSG implies that the effective volumetric gauge-length over which each strain value is computed is larger than the size of a single sub-set, with Equation 1 describing how the characteristic length of the VSG varies linearly with the aforementioned parameters [31].

$$
\begin{gathered}
L_{V S G}=\left(L_{S W}-1\right) * L_{S T}+L_{S S} \\
\text { Equation 1 }
\end{gathered}
$$

\subsection{Image processing}

The evaluation of the radius of curvature of the bent specimen was performed on the low magnification scan (4x) by using an industrial 3D metrology toolkit - GOM Inspect 2018 (GOM GmbH, Braunschweig, Germany [35]). As the approach requires inspection of polygonally meshed surfaces, a series of operations were carried out in Fiji ImageJ [36] and VGSTUDIO Max 2.5 (Volume Graphics $\mathrm{GmbH}$, Heidelberg, Germany [37]) respectively to export the greyscale based tomographs as a 2D meshed surface. Surface determination and the construction of a best fitting circle has been performed using the 'automatic' function, thus removing any user bias.

A second procedure was performed to check manually the result of the calculation described above. This was carried out in SOLIDWORKS 2015 (Dassault Systèmes SE, Vélizy-Villacoublay, France [38]), whereby the radius of curvature obtained through GOM Inspect 2018 was back-fitted onto the edge of the bent specimen. The workflow consisted of fitting a three-point arc (to the nearest pixel) to a 2D edge performed extraction. The flow-chart in Fig. 8 illustrates the process in detail. 


\section{Metrological approach}

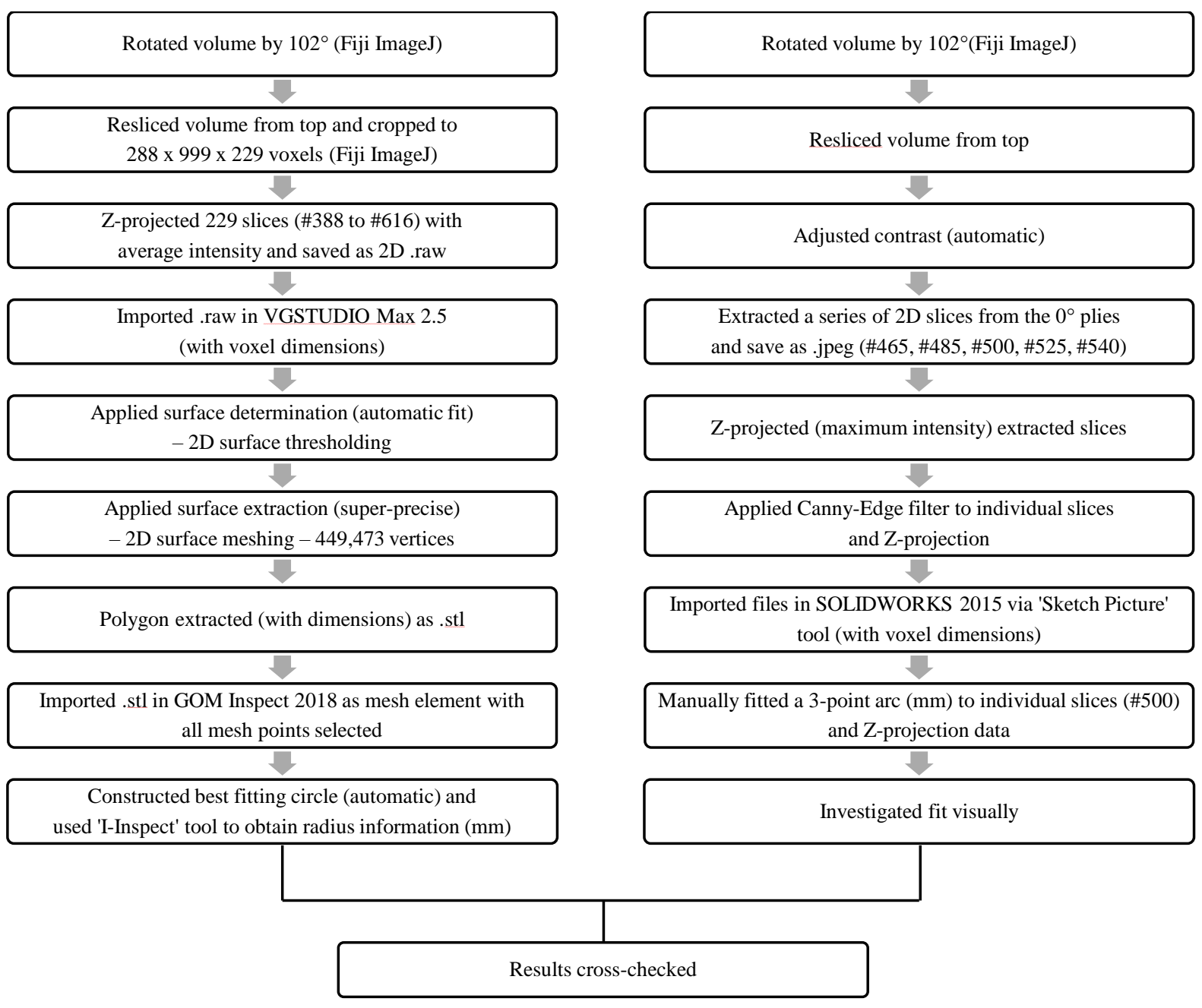

Figure 8 - Workflow illustrating the evaluation of radius of curvature of the bent specimen using GOM Inspect 2018 and validating the metrologically obtained results in SOLIDWORKS 2015.

\section{Results and discussion}

\subsection{Noise and sensitivity}

As noted above, to evaluate the metrological performance of the DVC technique, noise studies were conducted. The assessment involves two studies, with the approach being similar to that of Schöberl et al. [12], [13], Borstnar et al. [15], Gillard et al. [18] and Pierron et al. [20]. The first is a stationary analysis, which involves repeated scanning of the same specimen without any alterations in load (zero displacement, zero strain) or CT parameters (exposure, number of projections, beam energy, current or propagation distance). The purpose of this static noise test (SNT) is to quantify the intrinsic scanning noise and bias. The second study is a rigid body displacement (RBD) in which the specimen was moved in situ by a predefined displacement, parallel to the fibre direction (Y-axis), using the manipulator stage. CT acquisition was performed between each specimen position to evaluate the magnitude of the displacement vector fields and to assess any interpolation and/or system errors associated with the DVC approach [15]. 


\subsubsection{Static noise test}

Under ideal conditions, the obtained displacement vector data and implicit computed strains, should be equal to zero. Noise arising from the imaging system (e.g. photon counting statistics, electrical noise, scattered radiation) and the surrounding environment (e.g. induced vibrations, thermal changes) implies that this is never the case, and consequently sub-set displacements will generate false strains within the results [12], [13], [15], [18], [20]. However, due to the novelty of the DVC technique, as yet there is no agreed standard for the assessment of the strain error (usually referred to as the strain resolution) [15], [32]. For example, one approach reports the maximum standard deviation of a given strain map (plane of sub-sets) [18], [20], while a second returns the standard deviation of the measured strain components within the volume [12], [13], [15]. Following the first approach however can result in overestimating the error, as results can be affected by unrepresentative localised scanning artefacts (e.g. rings, detector 'zingers'). In this work, the latter approach is taken for which the error is seen to form a normal distribution with a mean of $\sim$ zero. The advantage of this approach is that both the stochastic noise in the imaging system and any biases in the correlation algorithm are captured and accounted for [12], [13], [15].

A typical effect of the sub-set size on the strain resolution is presented in Fig. 9. As previously reported in the literature, a compromise can be achieved between strain and spatial resolution [12], [13], [15], [17], [18], [19]. Of specific interest in this paper is the normal strain $\varepsilon_{y y}$, parallel to the fibre direction. Considering the normal components of the strain tensor, it can be observed that the lowest error is achieved in the fibre direction (Y), despite the fact that correlation along the fibres themselves has the lowest values, particularly in particle-free CFRPs [15]. This may be rationalised, as the deposition of $\mathrm{BaTiO}_{3}$ during the drum winding process occurred parallel to the fibre direction as the tow was drawn through the die; essentially creating longitudinal bands of fiducial markers in the Ydirection which assists the correlation process significantly, eliminating the self-similar microstructure. A similar effect, for the identical material, is also observed using Synchrotron Radiation Computed Tomography (SRCT) [12], [13].

The compromise between strain and spatial resolution occurs as a smaller sub-set size will contain too few unique features, in this case, the $\mathrm{BaTiO}_{3}$ particles. In contrast, a larger sub-set will compromise spatial resolution [39], as the underlying deformation is representative of the average displacement of all the voxels contained within the sub-set [15]. A larger sub-set size also implies a larger characteristic length for the VSG, which in turn has a negative impact on the global length-scale over which the strains are computed. Fig. 10 shows an isometric view of a typical sub-set, while Fig. 11 presents the number of particles as a function of sub-set size. As the fiducial markers are the main features used for correlating sub-sets between the different deformation states it may be assumed that matrix deformation dominates the DVC output [12], [13].

A trade-off is identifiable in Fig. 9 at an isotropic sub-set size of 120 voxels, where the corresponding length of the VSG measures 240 voxels. The equivalent VSGs (for different sub-set sizes applied) are shown in Fig. 12, alongside the strain resolution $\left(\varepsilon_{y y}\right)$ related to the static noise test. From Equation 1, it can be observed that for the particular case of a $50 \%$ sub-set overlap, the characteristic length of the VSG measures twice the size of the constituent sub-set dimension. 


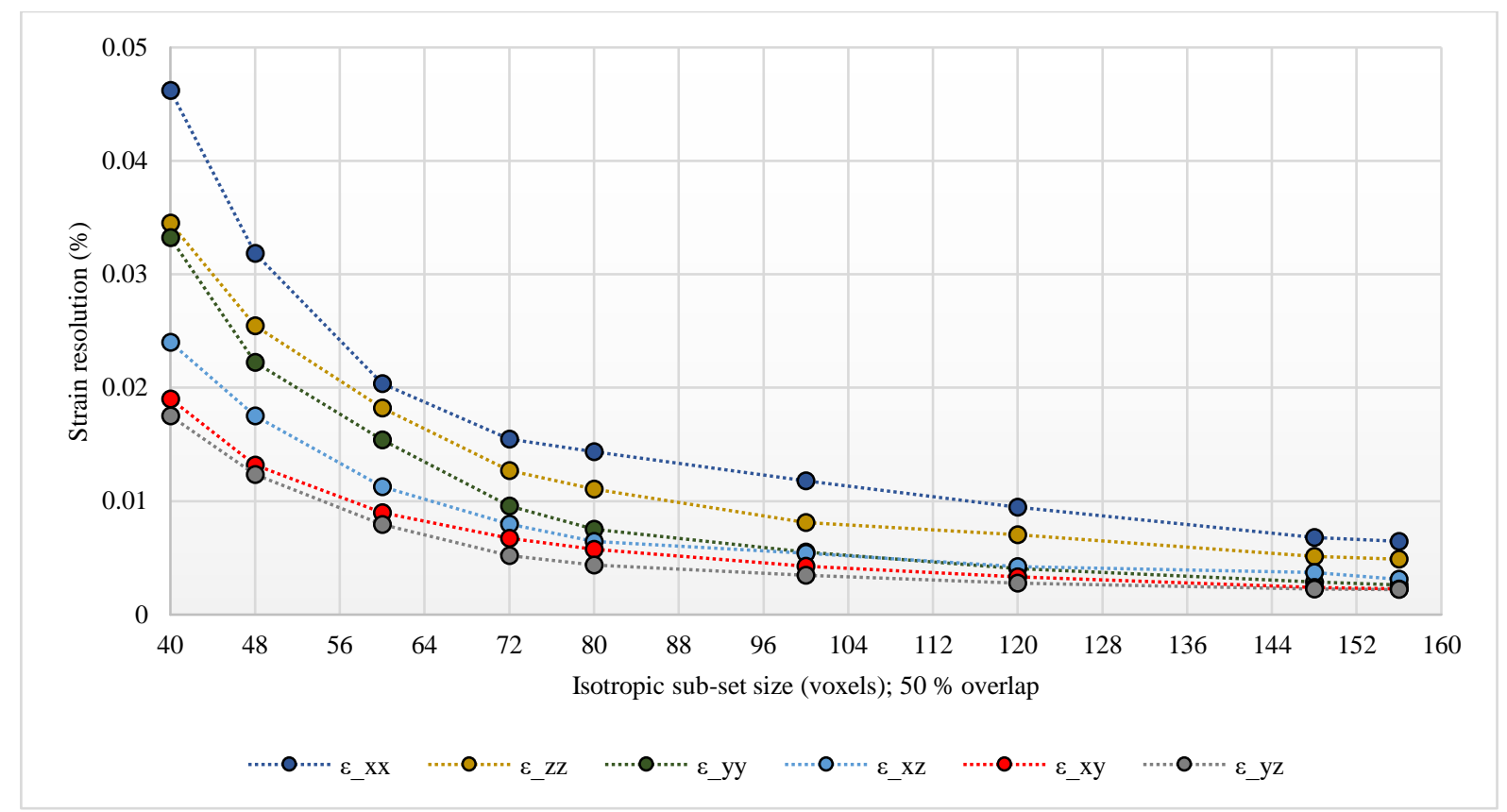

Figure 9 - Influence of sub-set size on the strain resolution during SNT scanning (expressed as the standard deviation of the measured strain values), illustrating the compromise between strain and spatial resolution. The $\varepsilon_{y y}$ component of the strain tensor denotes the longitudinal (fibre) strain, $\varepsilon_{x x}$ the in-plane transverse strain, while the out-of-plane strain component is given by $\varepsilon_{z z}$.

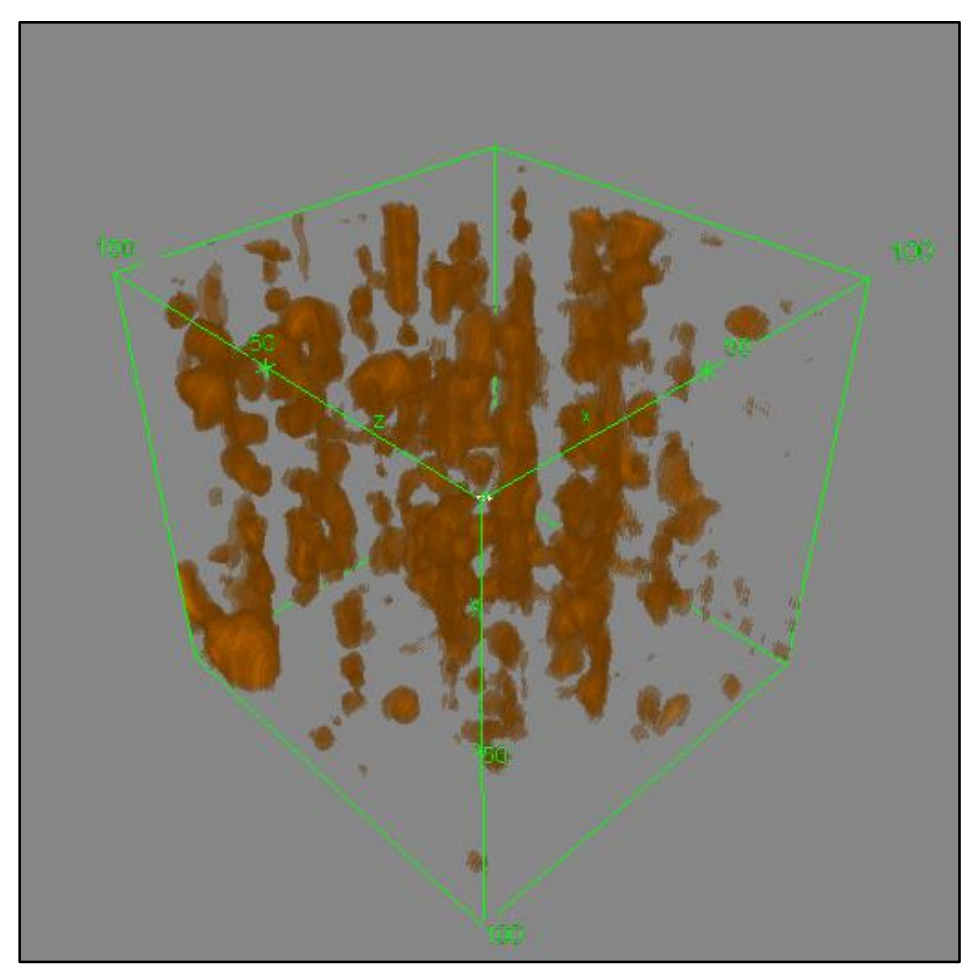

Figure $10-3 D$ isometric CT view of an isotropic sub-set with a characteristic length of 100 voxels, containing segmented $\mathrm{BaTiO}_{3}$ particles. 


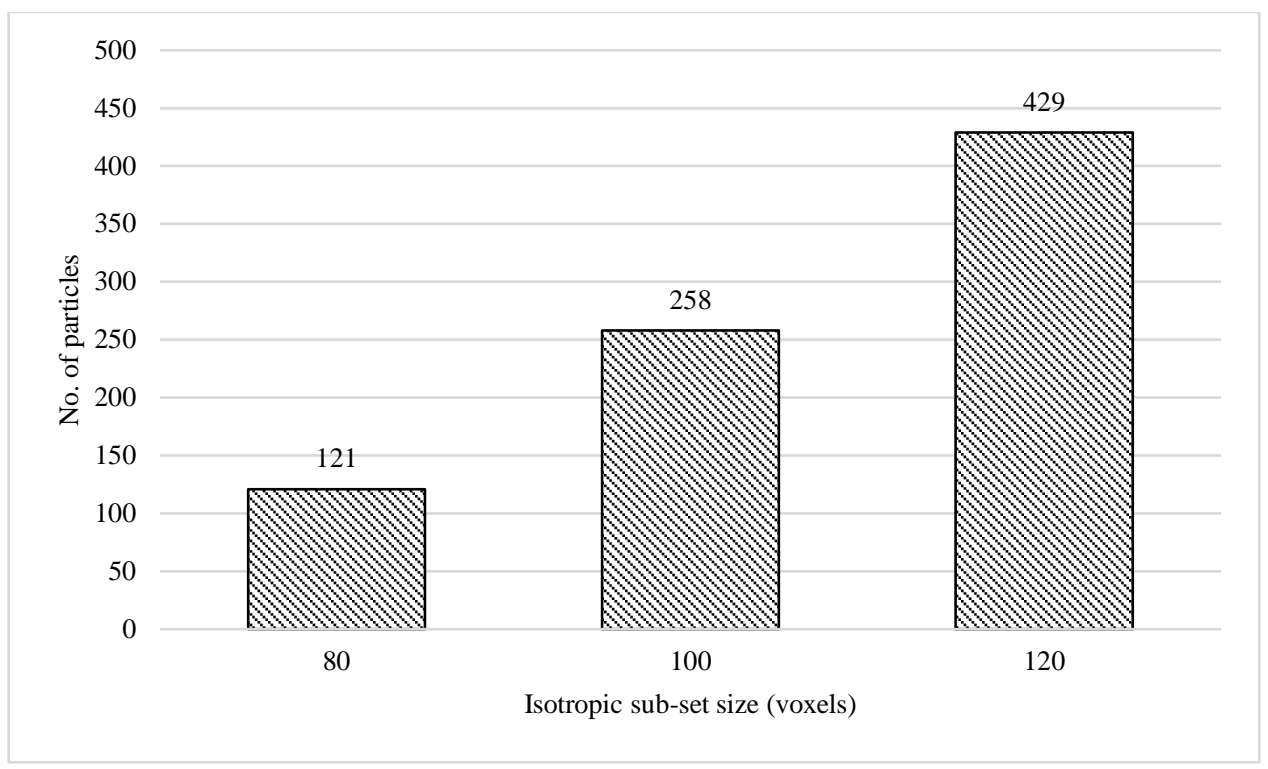

Figure 11 - Number of particles as function of sub-set size, using a grey-level threshold of 100/255.

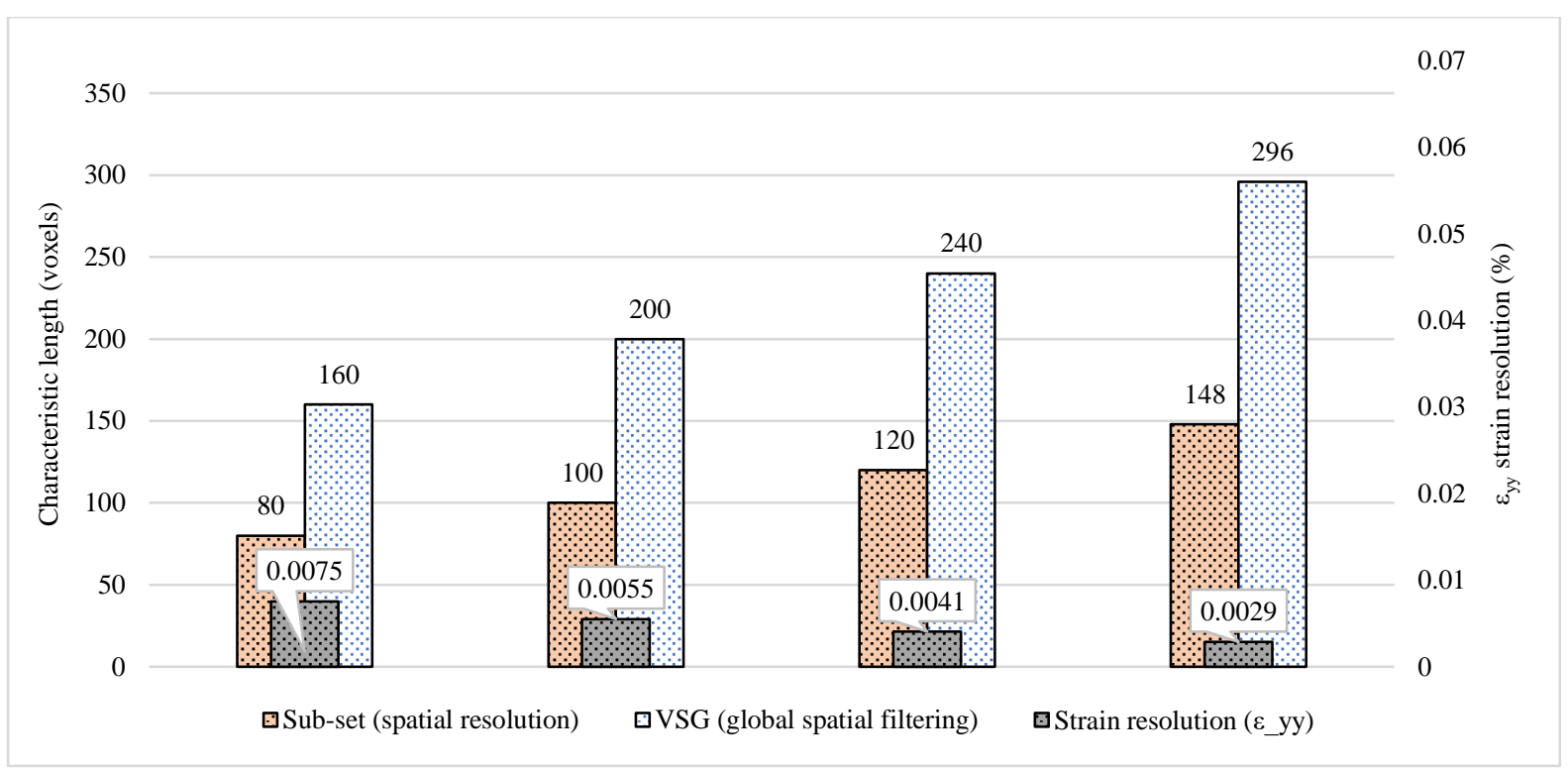

Figure 12 - Illustration of trade-off between VSG and sub-set size, in conjunction with spatial resolution vs. strain resolution $\left(\varepsilon_{y y}\right)$. Voxel size corresponds to $\sim 0.64 \mu \mathrm{m}$.

\subsubsection{Rigid body displacement}

The displacement field following a movement parallel to the fibre direction was initially computed at a sub-set size of 120 voxels (50\% overlap). The measured variation in displacement via DVC is relatively small, with an average displacement reading of $9.713 \mu \mathrm{m}$ and a standard deviation of 0.0148 $\mu \mathrm{m}$. The induced Y-displacement was manually confirmed to the nearest pixel in Fiji ImageJ; multiple measurements were taken by determining the distance between a unique feature (e.g. a void or $\mathrm{BaTiO}_{3}$ particle) and a reference point. The manual (average) measurement indicates a displacement of $\sim 9.62$ $\pm \sim 0.64 \mu \mathrm{m}$, which is within the accuracy of the DVC. As the present work is exclusively focused on 
fibre direction strain measurements, rigid body displacement analyses were limited to longitudinal tests. Although not an objective here, it is acknowledged that in order to perform reliable transverse (Xdirection), and out-of-plane (Z-direction) strain measurements additional noise assessment studies would be required (e.g. as in [15]).

A strain map indicating the rigid body correction is shown in Fig. 13, where the magnitude of the longitudinal strain noise is shown on a map overlapped with the microstructure. For smaller sub-sets, the SNT and RBD corrected errors $\left(\varepsilon_{y y}\right)$ are collectively summarized in Fig. 14. Edge-artefacts arising from volume displacements were geometrically masked prior to initiating the correlation process, measuring $795 \times 16$ invalid voxels (rounded to the closest integer), across each of the 230 raw data slices.

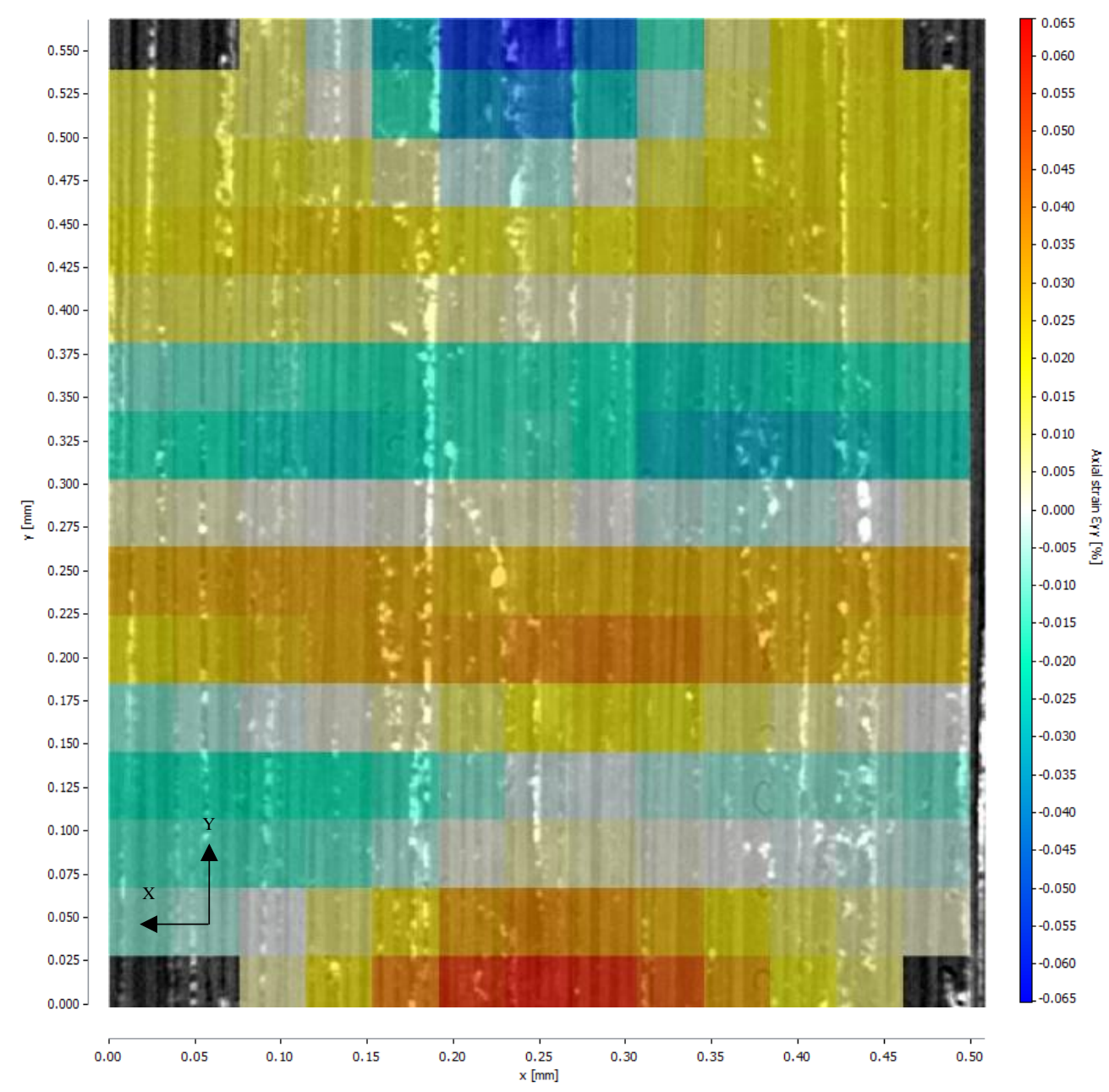

Figure $13-D V C$ slice ' $Z=2$ ' illustrating the $\varepsilon_{y y}$ strain map (noise) overlaid with the microstructure following RBD correction. Masking applied (795 x $16 \times 230$ voxels) to remove edge artefacts for the $Y$-displaced scans. Map range: -0.065 to $0.065 \%$. 

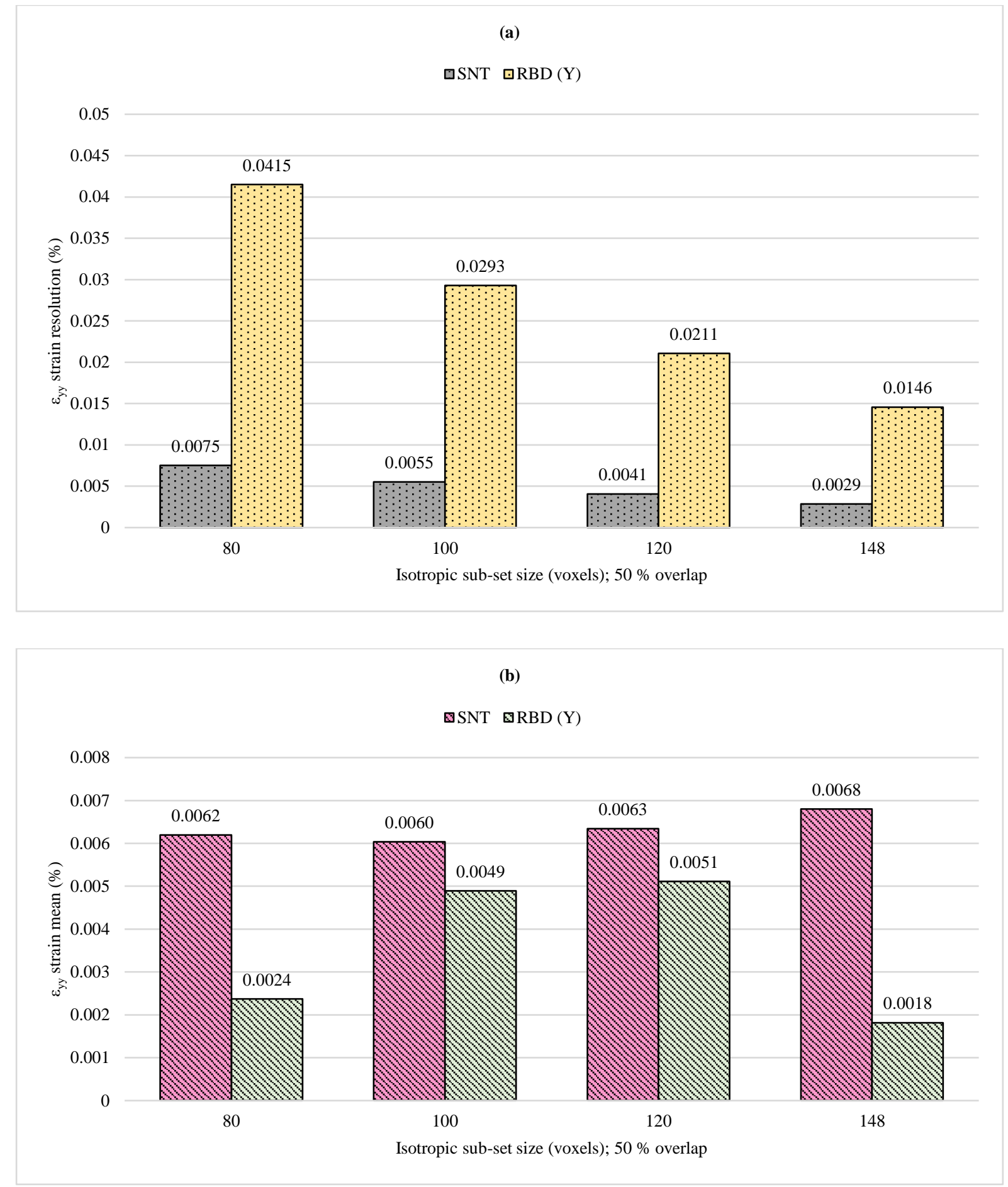

Figure 14 - (a) Strain resolution $\left(\varepsilon_{y y}\right)$, and (b) strain mean $\left(\varepsilon_{y y}\right)$ for the SNT and RBD corrected scans for various sub-set sizes with $50 \%$ overlap. Masking applied (795 $\times 16 \times 230$ voxels) to remove edge artefacts for the Y-displaced scans. Voxel size corresponds to $\sim 0.64 \mu \mathrm{m}$.

Fig. 14 (a) shows that in all cases, the RBD corrected error is higher than that of the SNT. This is consistent with previous studies which have shown that rigid body displacements can induce higher errors, particularly in the translation direction, due to additional errors from the interpolation biases arising from the specimen movement [15], [18], [20]. Based on the ex-situ configuration of the present experiment, the RBD strain resolution is considered as the major contribution to the experimental error. 
For the equivalent material and similar voxel resolution levels, but in the context of SRCT, it was shown that the discrepancies between the RBD corrected and SNT standard deviations for a given subset size are less than presented here [12], [13]. While it is not the aim of this paper to investigate the performance of the DVC based on different imaging acquisition methods, this is a potential indicator that the strain error following a rigid body displacement is particularly influenced by the interpolation function, which in turn is affected by the quality of the scan and/or acquisition parameters - e.g. noticeable reduced sharpness in volumes obtained through conventional lab CT (cathode tube) as opposed to SRCT in [12], [13].

Despite the different sources of radiation and dissimilar DVC algorithms/parameters (yielding different spatial resolutions), the magnitude of the RBD corrected strain resolutions are reasonably consistent with those reported in [12], [13] using $\mathrm{BaTiO}_{3}$ filled CFRPs and in particle toughened interlayer materials [15].

\subsection{Evaluation of radius of curvature}

The automatically generated radius of curvature generated in GOM Inspect 2018 is shown in Fig. 15, where a close-up view of the best-fitted circle can be visualized. The obtained radius measures 65.38 $\mathrm{mm}$ or the equivalent of $\sim 16,263$ voxels $(\sim 4.02 \mu \mathrm{m}$ voxel size $)$. A key observation can be readily made regarding the dimension of the circle, which is large in relation to the geometry of the specimen captured within the FOV of the $4 \mathrm{x}$ scan. This, however, follows the above described methodology of the experiment whereby a small deflection was intended in order to maintain compatibility with the classical beam theory as well as to avoid any microstructural damage within the $0^{\circ}$ plies.

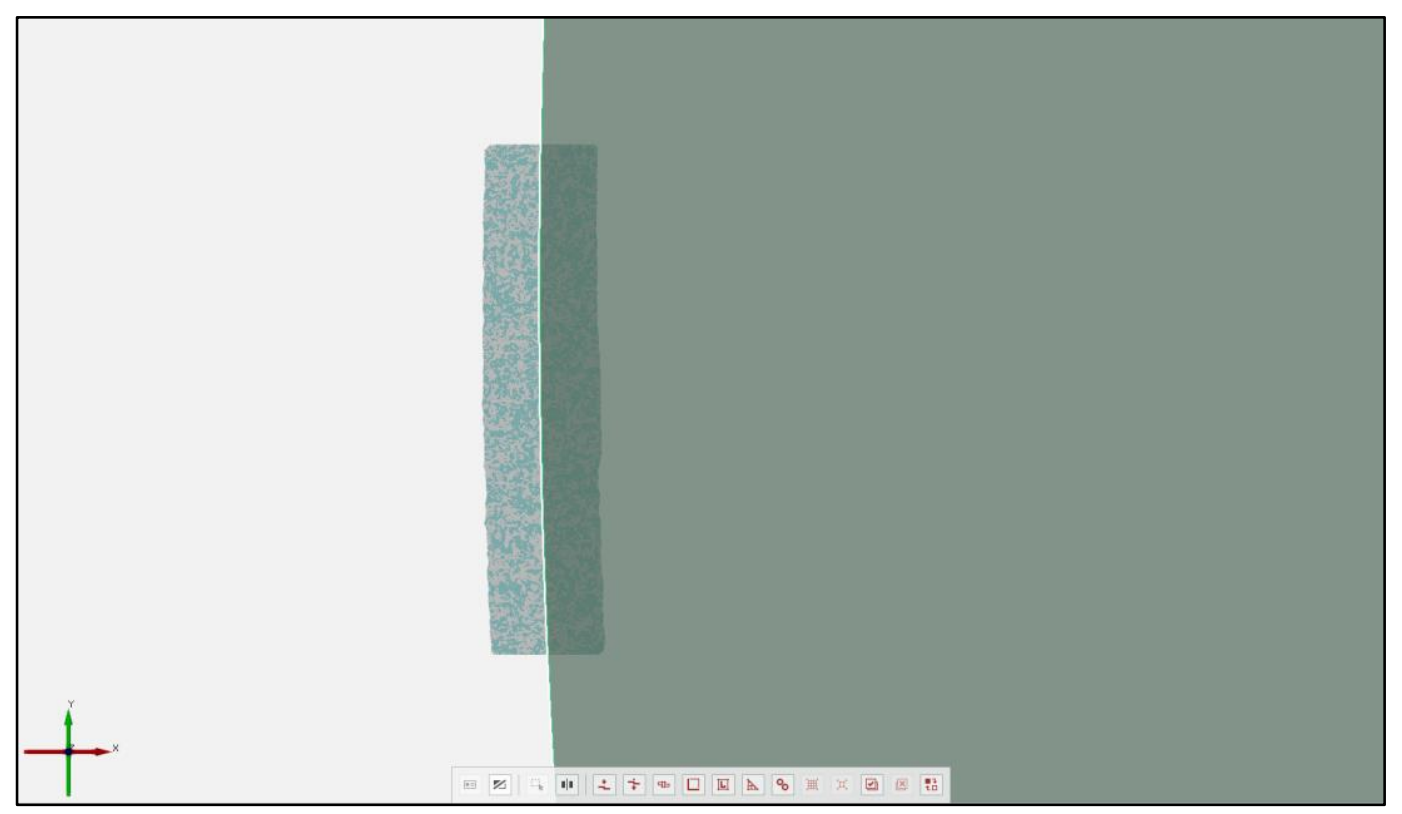

Figure 15 - Metrologically fitted circle with a radius of $65.38 \mathrm{~mm}$ on the specimen bent geometry $(\sim 1 \mathrm{~mm} x \sim 4$ mm) captured within the FOV of the $4 x$ scan.

Following the workflow earlier described in Section 2.5., this result was manually back-fitted onto the specimen edge in SOLIDWORKS 2015. A good agreement was found between the two techniques, and therefore the data generated by GOM Inspect 2018 is considered correct. The value of $65.38 \mathrm{~mm}$ is thus used in the evaluation of the strain profiles as function of the specimen thickness (Section 3.3.). 


\subsection{Beam strain profiles}

For an ideally homogeneous material, it is expected that deformation under elastic bending would yield a smooth linear gradient about the central neutral axis of the specimen. Divergence from this may be attributed to experimental errors (stochastic, systematic etc.) and/or variations in microstructure (e.g. local fibre misalignment).

Once the 3D grid of centroid displacements was numerically differentiated to produce full-field 3D strain maps, the constituent sub-sets can either be evaluated as standalone or averaged with the neighbouring sub-sets in various post-processing schemes for comparison purposes. An exemplar averaging scheme is presented schematically in Fig. 16, initially carried out on a volumetric basis (through the entire DVC stack), followed by the analysis of an individual DVC slice - see Table 2 and Fig. 7. As such, for a centroid position along the X-axis, a column of sub-sets (Y-direction) was averaged both in the in-plane and in the out-of-plane direction $(\mathrm{Z})$. Subsequently, the process was repeated incrementally for all permutations in the X-direction. For the grid obtained through a correlation with a sub-set size of 120 voxels with $50 \%$ overlap (Fig. 16), each of the columns contain 15 sub-sets, and spans four DVC slices (planes) in the Z-direction - thereby representing an average of 60 sub-sets (with the exception of the corners of the overall volume, due to cropping/making, where the total number of sub-sets per column are slightly less). Finally, each of the 13 averaged columns was compared against the Euler-Bernoulli prediction. The theoretical values, corresponding to the reciprocal of the radius of curvature $(65.38 \mathrm{~mm})$, were computed at the position of each sub-set column on the grid. The planar procedure is similar, with the only difference being that sub-set averaging was performed independently in each of the slices (i.e. limited to the in-plane direction: 15 sub-sets were averaged in one instance, as opposed to 60 for across whole volume). Next, the averaging scheme followed a decomposition into blocks comprised of a reduced number of sub-sets: 3 blocks of 5 subsets followed by 5 blocks of 3 sub-sets per each column, corresponding to a sub-set sampling of 33.33 $\%$ and $20 \%$ respectively per column. Ultimately, the assessment was carried based on standalone subsets (multiple rows) through the thickness of the beam. 


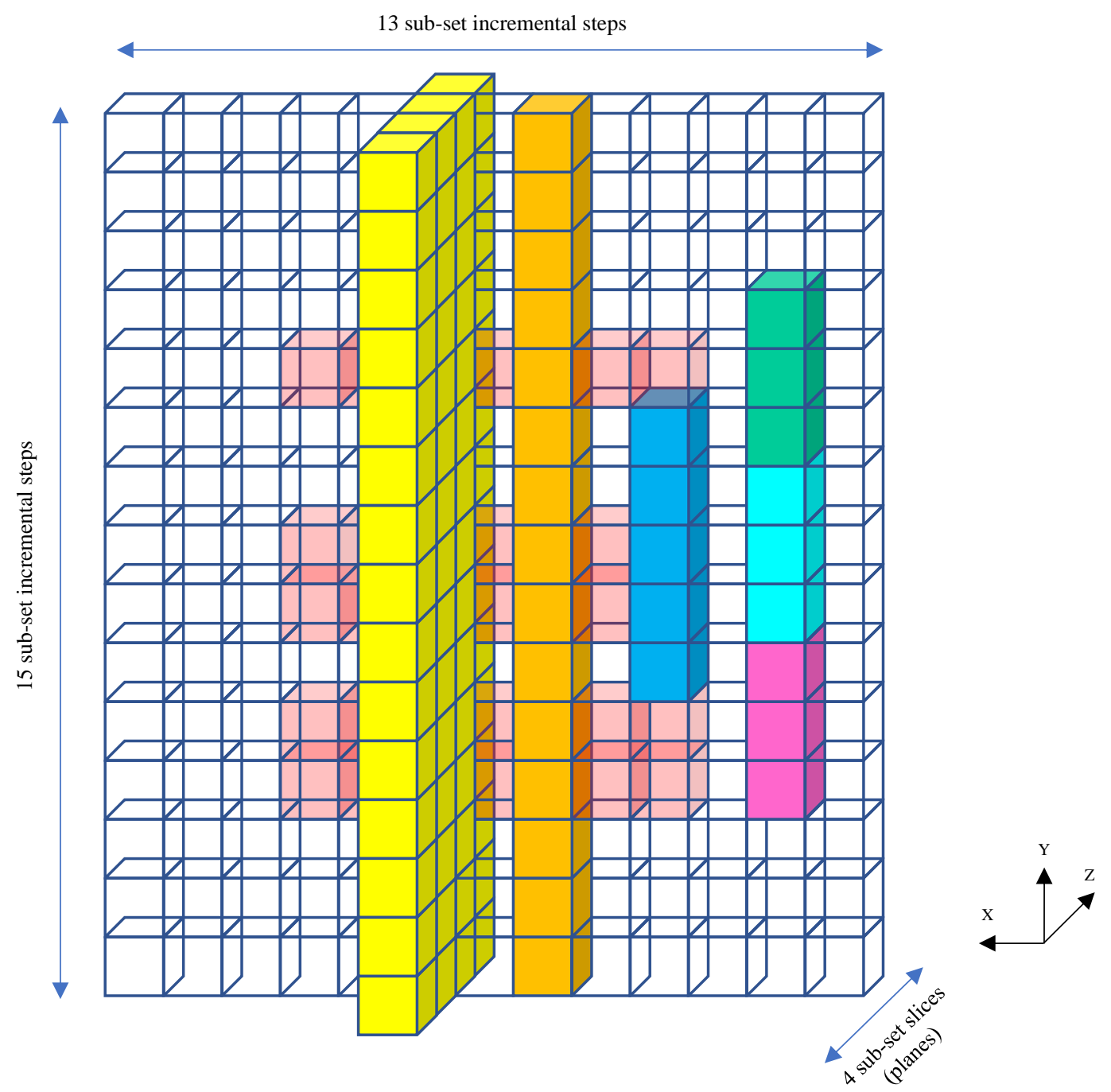

Figure 16 - Schematic diagram illustrating the 3D grid of DVC sub-sets, applicable to matching volumes of 795 $x 885 x 230$ voxels, correlated with an isotropic sub-set size of 120 voxels and $50 \%$ overlap. For simplicity,

only overlapping windows (steps) are shown as opposed the grid comprised of overlapped sub-sets.

Displacement vectors, and implicit strain values are computed for each step (i.e. a distance of 60 voxels or

$\sim 38.4 \mu \mathrm{m}$ ). The sub-set averaging schemes are indicated as follows in decreasing stages (applicable to each permutation along the X-direction: yellow - volumetric across four slices (planes) of sub-sets, orange - planar, across a single slice of sub-sets, blue - planar, single median block of five sub-sets '2/3', pink, cyan and green - planar, three central blocks each consisting of three sub-sets '2/5', '3/5', '4/5' and red-planar, standalone sub-sets (rows) through the beam thickness compared with the analytical solution.

A typical strain profile obtained through the four-point experiment can be seen in Fig. 17, in which the microstructure is overlaid with the strain map. The expected strain distribution can be observed qualitatively through the beam thickness, i.e. fibres in tension of the left-hand side and in compression on the right-hand side, with a neutral plane ( zero strain) located centrally. 


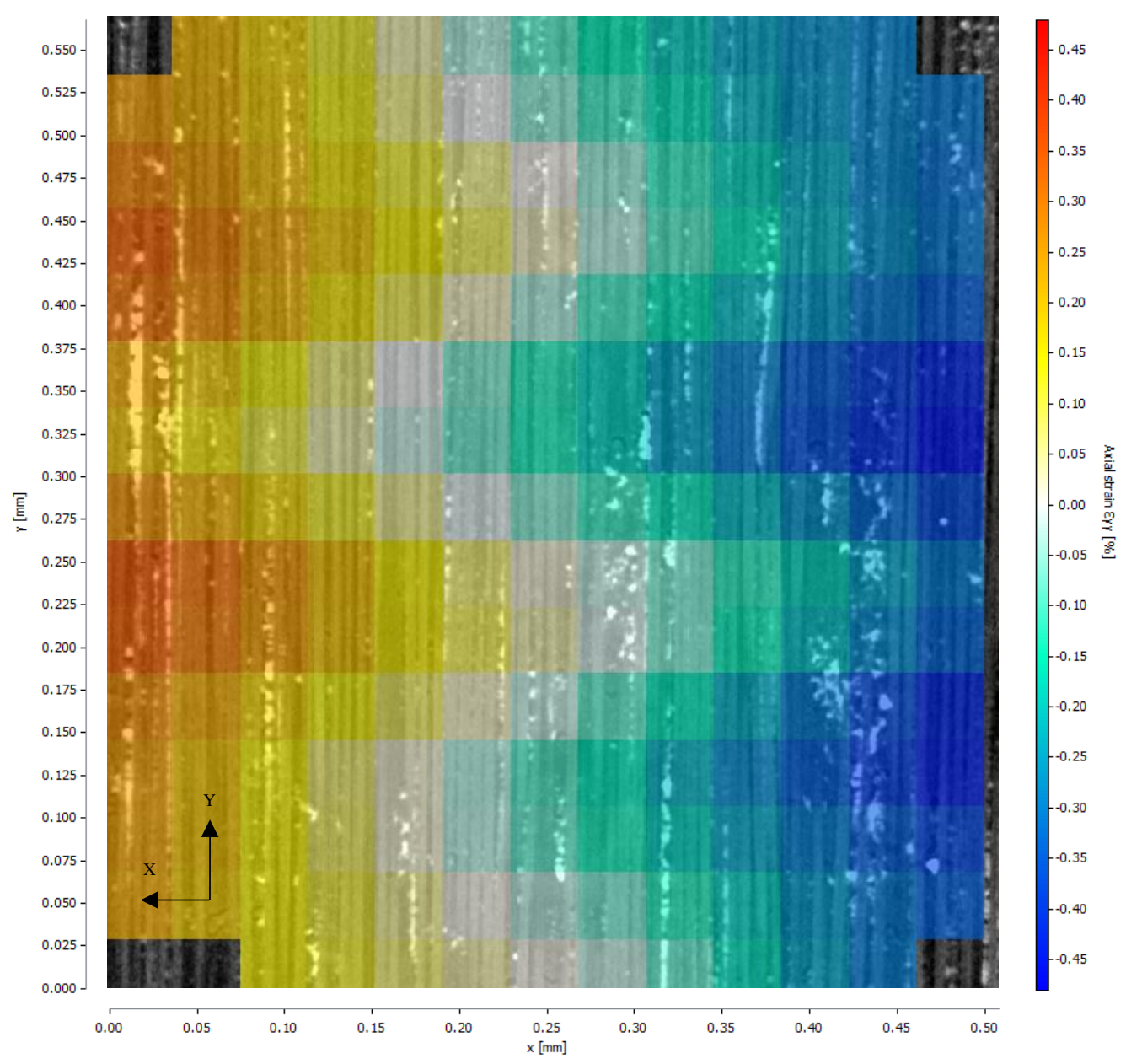

Figure 17 - Strain mapping illustration overlapped with the microstructure (795 $x 885$ voxels, 0.645 voxel size), showing the through-thickness strain distribution. Fibres in tension of the left-hand side and in compression on the right-hand side. Sub-set size: 120 voxels with $50 \%$ overlap. DVC slice number ' $Z=2$ '. Interpolation/smoothing not applied to the strain map.

\subsubsection{Volumetric sub-set averaging}

Fig. 18 (a) presents the strain data obtained from a correlation based on sub-set size of 120 voxels with $50 \%$ overlap. Similarly, Fig. 18 (b) and Fig. 18 (c), present the volumetrically averaged strain gradient, but at different spatial resolutions (100 voxels and 80 voxels sub-set size respectively). As expected, the spatial sampling improves with decreasing sub-set size, but with an increased predisposition to noise - see Table 3 and Fig. 9.

Following the volumetric averaging scheme of sub-sets, the DVC output is found to be in concordance with the analytical solution for all three presented case studies. Even though the experimental error doubles $(0.0415 \%$ vs. $0.0211 \%)$ from a sub-set size of 80 voxels to 120 voxels, the strain gradient is shown not to be affected. 
(a)
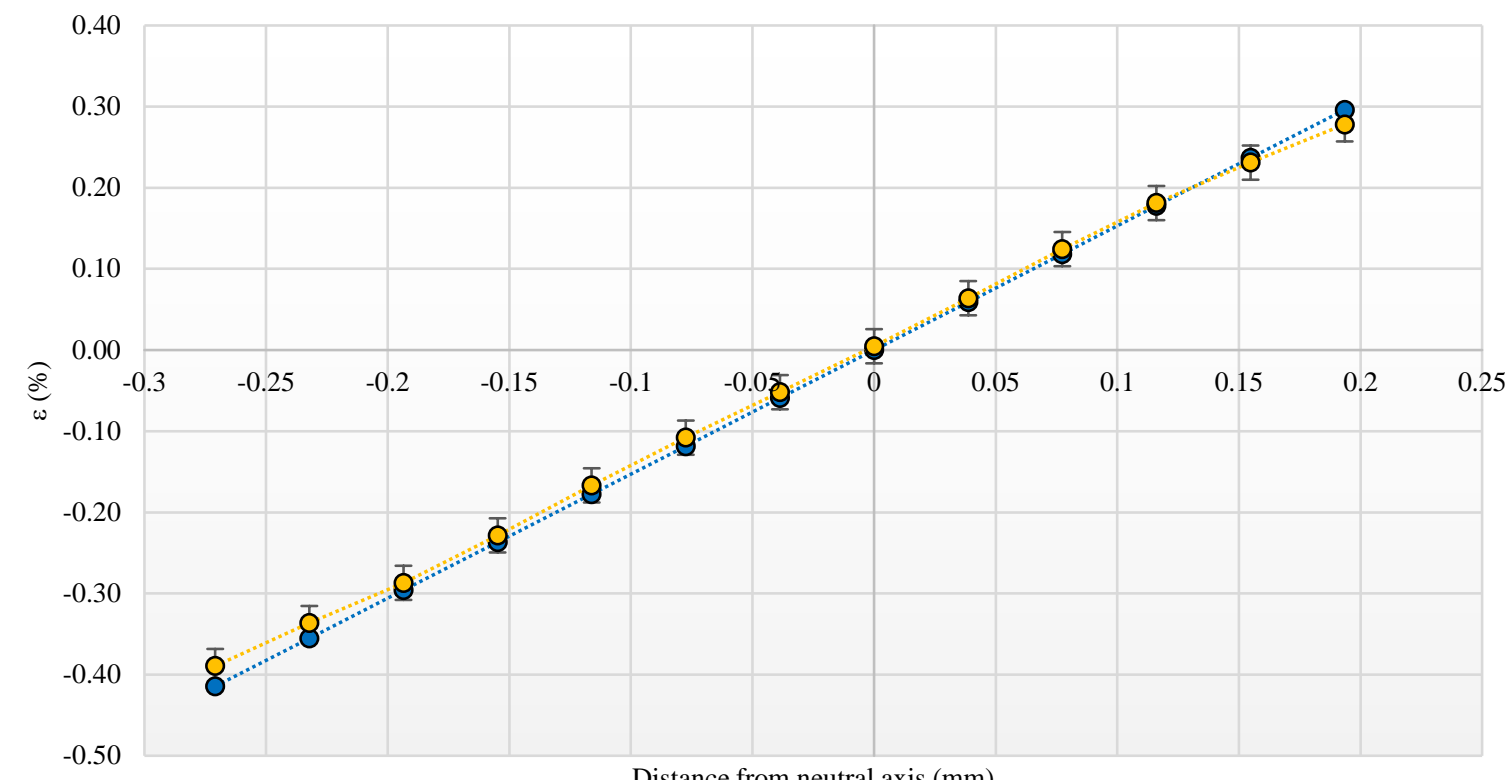

Distance from neutral axis (mm)

........ Euler-Bernoulli

- -... DVC for $\mathrm{Z}=0-3$ (120 voxels, $50 \%$ overlap)

(b)

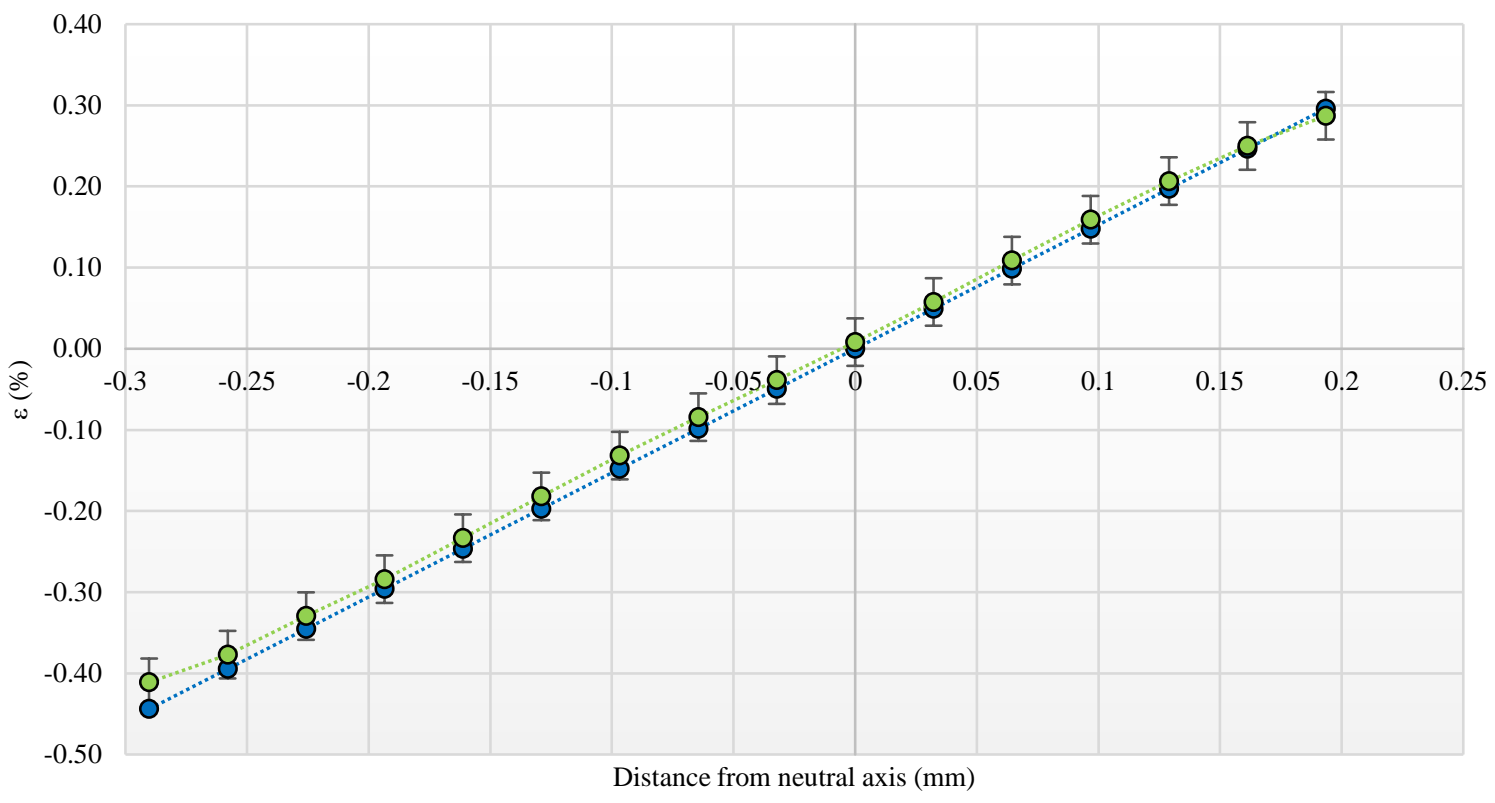

$\ldots \ldots$ Euler-Bernoulli $\quad \cdots \cdots$ O..... DVC for $\mathrm{Z}=0-4$ (100 voxels, $50 \%$ overlap) 
(c)

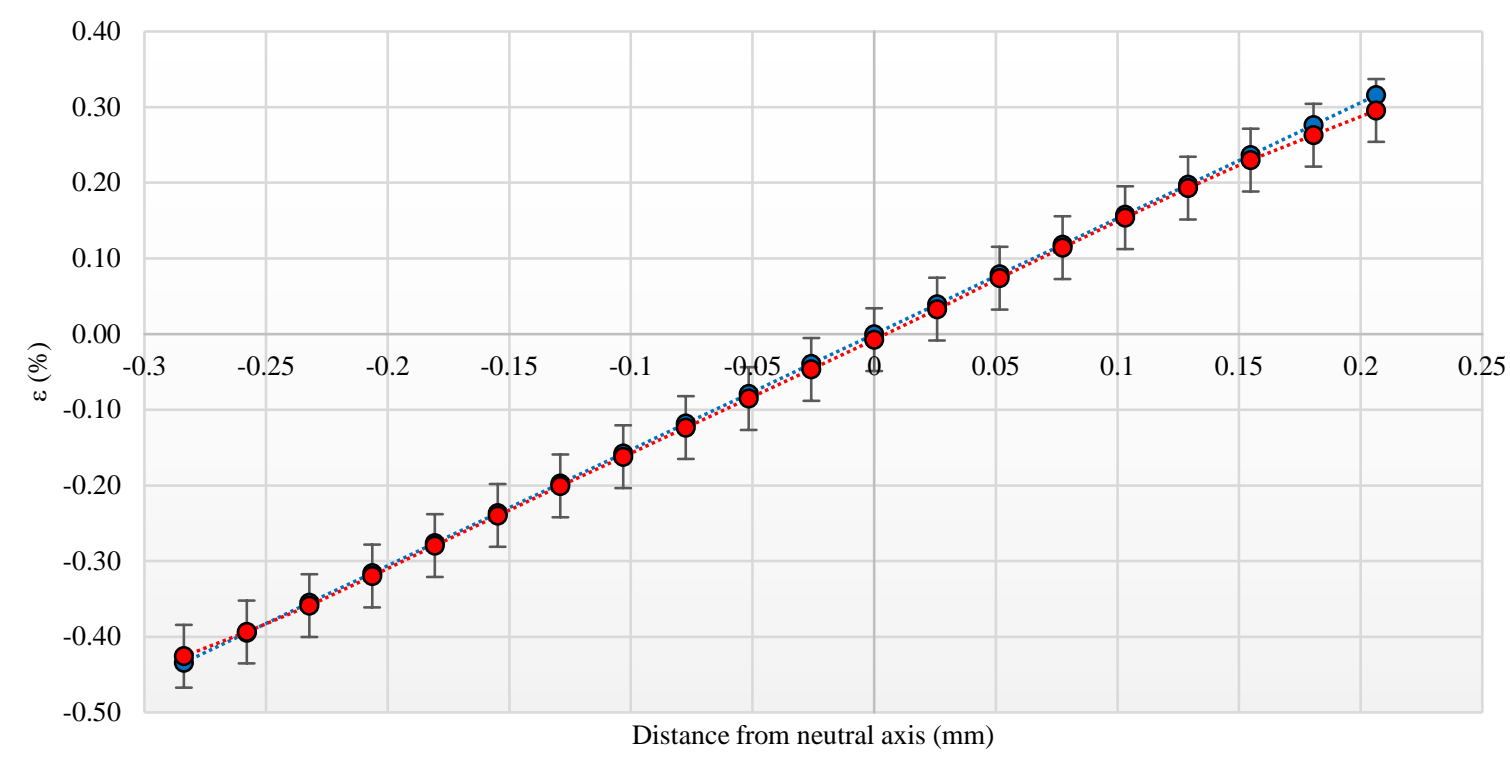

…..... Euler-Bernoulli $\quad$......... DVC for $\mathrm{Z}=0-5$ (80 voxels, $50 \%$ overlap)

Figure 18 - Strain gradient obtained through the application of DVC vs. classical beam theory prediction. Subset size: (a) 120 voxels, (b) 100 voxels, (c) 80 voxels with $50 \%$ overlap. Volumetric sub-set averaging through all DVC slices: (a) ' $Z=0$ ' to ' $Z=3$ ', (b) ' $Z=0$ ' to ' $Z=4$ ', (c) ' $Z=0$ ' to ' $Z=5$ '. Number of averaged sub-sets per column: (a) 60 (15 x 4), (b) 72 (18 x 4), (c) 88 (22 x 4). Number of data points: (a) 13 columns, (b) 16 columns, (c) 20 columns. Indicated RDB corrected error of (a) $0.0211 \%$, (b) $0.0293 \%$, (c) $0.0415 \%$.

A key observation from Fig. 18, is related to what appears to be an offset of the neutral axis by 77.4 $\mu \mathrm{m}$ to $96.75 \mu \mathrm{m}$ (depending on the sub-set grid spacing), leading to a higher number of data points in compression as opposed to the tension side of the beam, across all examples (e.g. 7 data points in compression vs. 5 data points in tension from the neutral axis, for the 120 voxel sub-set size case, with $50 \%$ overlap). This effect is a direct experimental artefact arising from image acquisition, and not DVC processing, whereby the CT volumes of the deformed and undeformed state were slightly offset in relation to the geometrical neutral axis of the specimen. The reduced FOV at 20x magnification made a perfect alignment at fibre-level challenging, which combined with the offset required to implement an eccentric centre of rotation relative to the mid-axis of the rig for the bent configuration, resulted in matching volume images marginally orientated towards the compression side of the beam.

In addition, from Fig. 18, a small degree of nonlinearity can be observed at the furthest distance from the neutral axis (corresponding to the highest negative/positive strain values within the FOV). This effect is consistent throughout the data sets, and can be attributed to edge-based artefacts - see Section 2.4. The effect is confined to three grid positions nearest the edge of the FOV, which is the equivalent to the span of the VSG. This is anticipated, as a poorly correlated sub-set positioned at the edge of the matching volumes will have a detrimental impact on the entire corresponding VSG measurement of strain. Further DVC results have been truncated to eliminate these edge-artefacts located at the perimeter of the correlated volumes (XY-plane), at the expense of a reduced number of data points through the beam thickness (Section 3.3.2.). It can also be observed, that as the sampling rate is increased (i.e. smaller sub-set size/higher spatial resolution), the gauge-length of the affected area diminishes, confirming that this is an artefact of the image processing. 
Following the above described volumetric sub-set sampling and averaging strategy, the effectiveness of DVC in measuring local strains is shown to correlate well with Euler-Bernoulli beam theory, however with a subtle reduced accuracy in the presence of edge-artefacts.

\subsubsection{Planar sub-set averaging}

For brevity, correlation results in this section are based on a sub-set size of 120 voxels (50\% overlap), with averaging now being carried out in a single slice (plane) of the specimen. In the first instance single-sliced columns of sub-sets are considered as opposed to columns averaged through the overall stack. Fig. 19 shows strain gradients obtained through the decomposition of the DVC stack into the component DVC slices. Whilst there is a reduction in the number of sub-sets averaged per data point (column) by a quarter, i.e. from 60 to 15 , the linearity of DVC output remains in good agreement with the analytical solution for the middle DVC slices shown here ' $\mathrm{Z}=1$ ' and ' $\mathrm{Z}=2$ '. As noted in the previous section, the number of data points through the thickness of the specimen was reduced to eliminate edgebased artefacts.

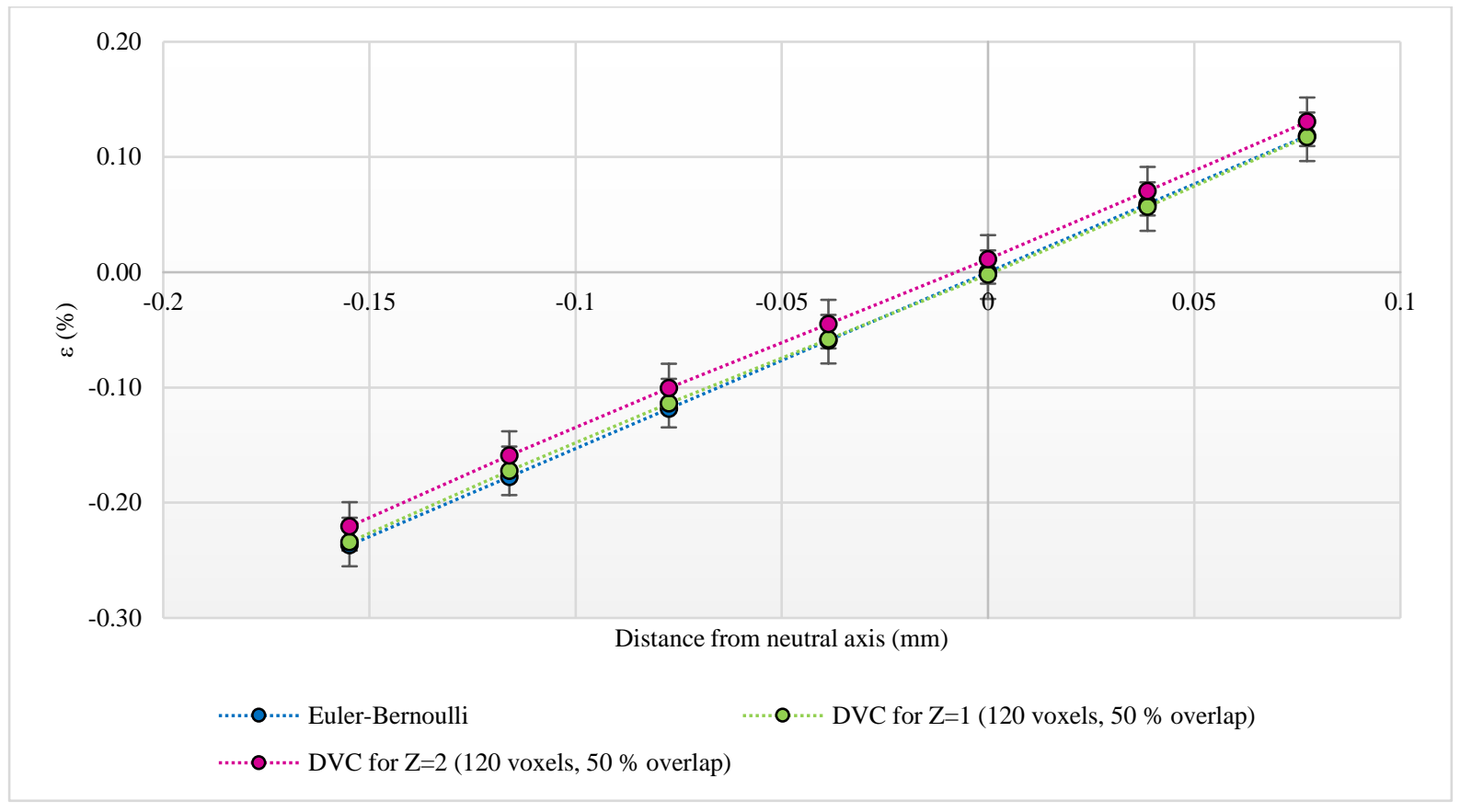

Figure 19 - Strain gradient obtained through the application of DVC vs. classical beam theory prediction. Subset size: 120 voxels with $50 \%$ overlap. Planar sub-set averaging performed independently in both DVC slices ' $Z=1$ ' and ' $Z=2$ '. Number of averaged sub-sets per column: 15 (15 $x 1)$. Edge-artefacts located at the perimeter truncated (XY-plane), yielding 14 data points (7 columns $x 2$ DVC slices). Indicated RDB corrected error of $0.0211 \%$.

The two remainder DVC slices ' $\mathrm{Z}=0$ ' and ' $\mathrm{Z}=3$ ' are not discussed, as comparable to what was stated in Section 3.3.1., these planes of sub-sets also represent edge-artefacts, not being fully satisfied from a voxel perspective. However, in this particular instance, the outlier sub-sets are not positioned at the perimeter of the grid (XY-plane) for each DVC slice, but are rather located at the top/bottom end of the 3D stack (Z-direction) - see Section 2.4. Consequently, due to missing pixel information, restoring the 'true' response of the material is more challenging in the aforementioned DVC slices.

Further decomposition of the averaging scheme concentrates on the results based on a single DVC slice, in particular slice ' $\mathrm{Z}=2$ '. Blocks are considered, being comprised of a reduced number of sub-sets 
(as opposed to full single-sliced columns) and remote from edge-artefacts: median block number ' $2 / 3$ ' comprised of 5 sub-sets - Fig. 20 (a), and central blocks ' $2 / 5$ ', ' $3 / 5$ ', ' $4 / 5$ ' each comprised of 3 sub-sets - Fig. 20 (b). Finally, the averaging scheme is removed completely, and an example is shown where single rows of sub-sets are compared against the theoretical prediction - Fig. 21.

Decreasing the number of averaged sub-sets in a column even further, from 15 to 5 , is also shown not to introduce fluctuations above the experimental error in the strain gradient - Fig. 20 (a). The level of nonlinearity is observed to increase slightly when only three sub-sets are averaged per column, as opposed to a number of five, but the DVC output still remains within the boundaries of the RBD corrected error of $0.0211 \%$ - Fig. 20 (b). 
(a)
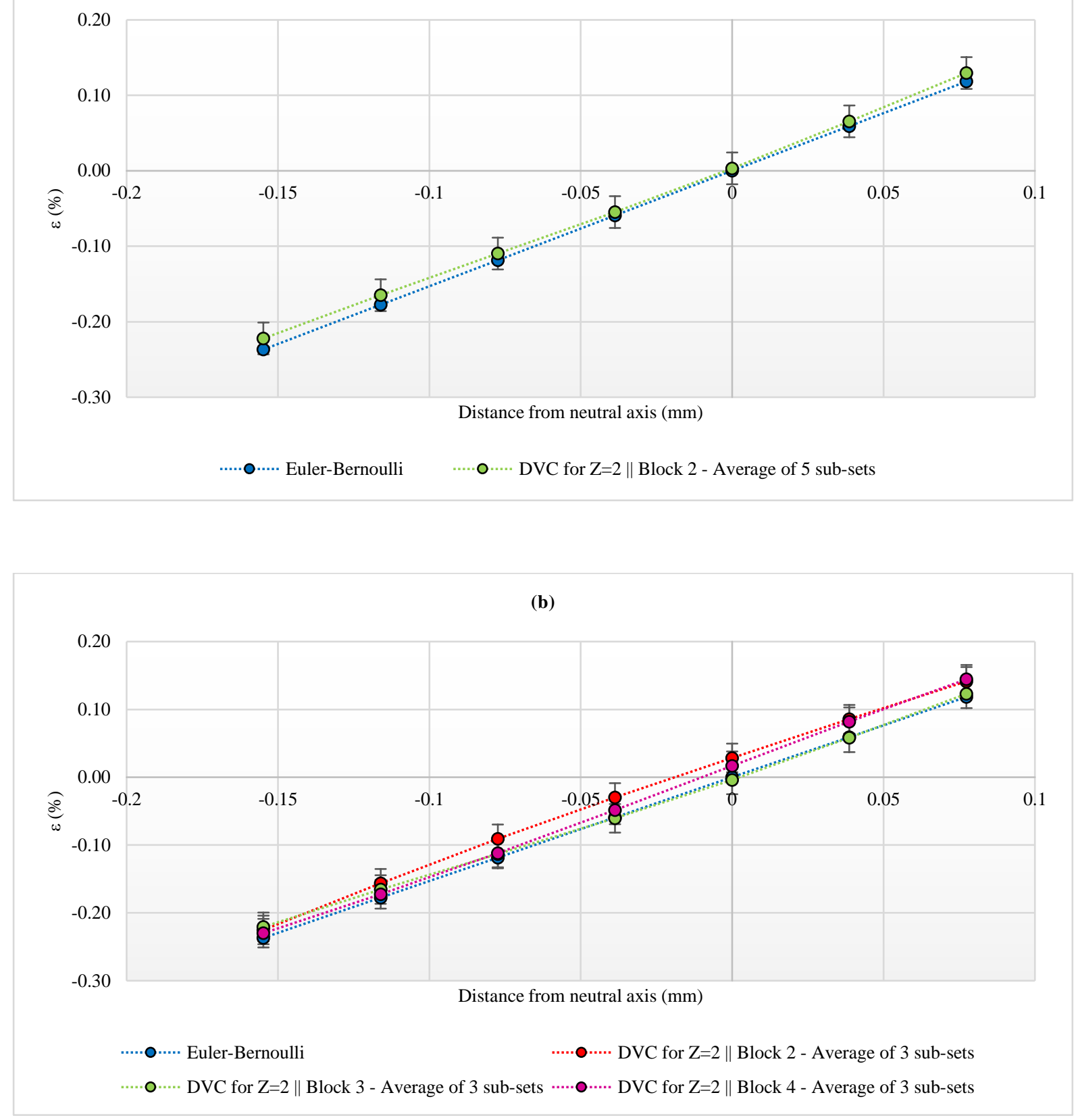

Figure 20 - Strain gradient obtained through the application of DVC vs. classical beam theory prediction. Subset size: 120 voxels with $50 \%$ overlap. DVC slice number ' $\mathrm{Z}=2$ '. Planar averaging scheme: (a) single median block '2/3' containing 5 averaged sub-sets per column on the grid (block contains $33.33 \%$ of the total number of sub-sets per column), and (b) central blocks '2/5', '3/5', '4/5' each containing 3 averaged sub-sets per column on the grid (one block contains $20 \%$ of the total number of sub-sets per column). Edge-artefacts located at the perimeter and ends of the stack truncated (XY and Z-plane), resulting in: (a) 7 data points ( 1 block of averaged sub-sets $x 7$ columns), and (b) 21 data points (3 blocks of averaged sub-sets $x 7$ columns). Indicated RDB corrected error of $0.0211 \%$. 


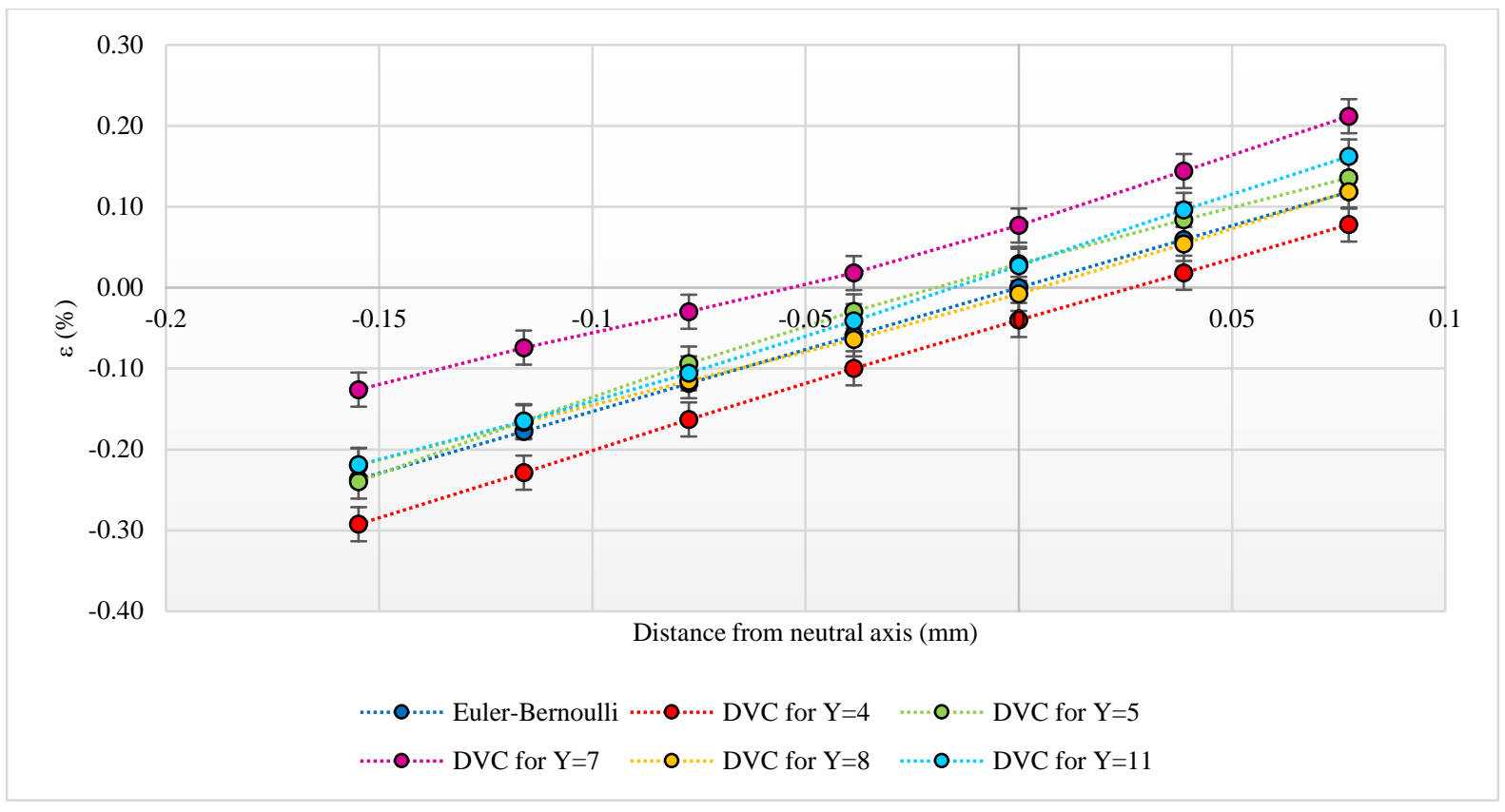

Figure 21 - Strain gradient obtained through the application of DVC vs. classical beam theory prediction. Subset size: 120 voxels with $50 \%$ overlap. Sub-set averaging scheme removed (i.e. each data point corresponds to strain value of a standalone sub-set). Rows of sub-sets (Y-direction) plotted: 4/15, 5/15, 7/15, 11/15, resulting in 28 data points (7 sub-sets $x 4$ rows). Edge-artefacts located at the perimeter and ends of the stack truncated (XY and Z-plane). Indicated RDB corrected error of $0.0211 \%$.

Sampling standalone sub-sets with a size of 120 voxels $(\sim 76.8 \mu \mathrm{m}$ or $\sim 10$ fibre diameters), as opposed to averaging entire columns or reduced blocks comprising multiple sub-sets, does reveal strain values through the beam thickness in which the nonlinearity and/or offset exceeds that of the computed experimental error (Fig. 21). No conclusive explanation can be drawn as to why a deviation from the Euler-Bernoulli prediction occurs at such relatively small length-scales, but it is likely that this represents the practical limit of the current technique, linked to the effective sampling of the beam. More specifically, the eccentric centre of rotation relative to the mid-axis of the rig for the bent configuration could have resulted in tomographic reconstructions with underlying:

- Different attenuation profiles, due to varying X-ray path lengths

- Oversampled/undersampled radiographs of the central gauge and edge region.

Additionally, the deviation may also be related to strain variations associated with local changes in the material microstructure, including fibre misalignment (fibre direction errors and waviness), fibrematrix volume fraction fluctuations, as well as a nonuniform distribution of $\mathrm{BaTiO}_{3}$ fiducial markers within the fibrebed. Raw CT data slices shown in Fig. 22 illustrate that the fabricated material presents such local microstructural changes. Voids represent an additional variability; however, the implications are presumed second-order due to a relatively dispersed distribution within the CFRP.

Maintaining the overall argument that the experimental data agrees with the theoretical prediction, and that the effectiveness of the technique is validated, additional studies via DVC in conjunction with $\mathrm{CT}$ are envisaged within the $0^{\circ}$ plies (not limited to elastic deformations): matrix plastic yielding, fibrematrix interfacial debonding, longitudinal matrix microcracking, inter/intra-laminar delaminations etc. Furthermore, the technique can be employed to orientations different from the $0^{\circ}$ plies $($ e.g. transverse matrix cracking), as well as in different loading scenarios (e.g. compression, torsion) and materials with multiaxial or discontinuous reinforcement. 


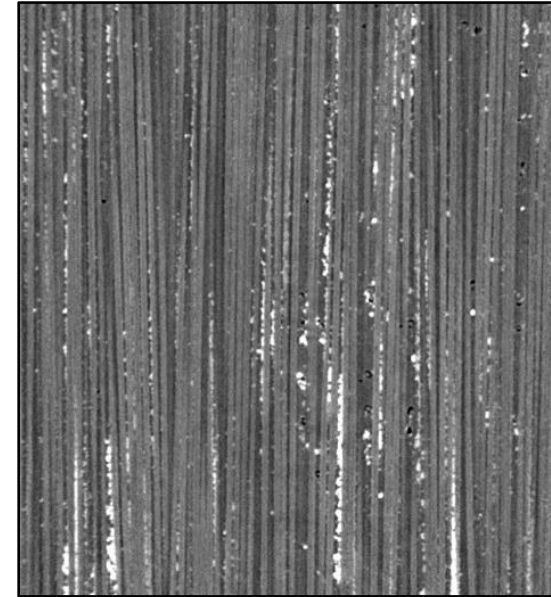

(a) Raw CT slice $98 / 230$

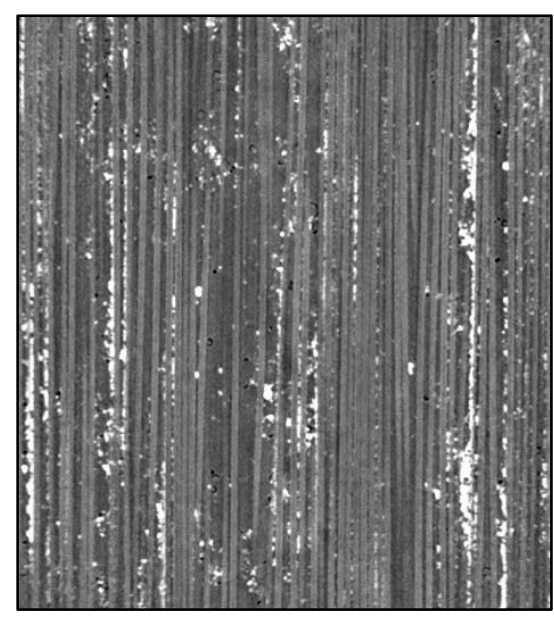

(c) Raw CT slice $162 / 230$

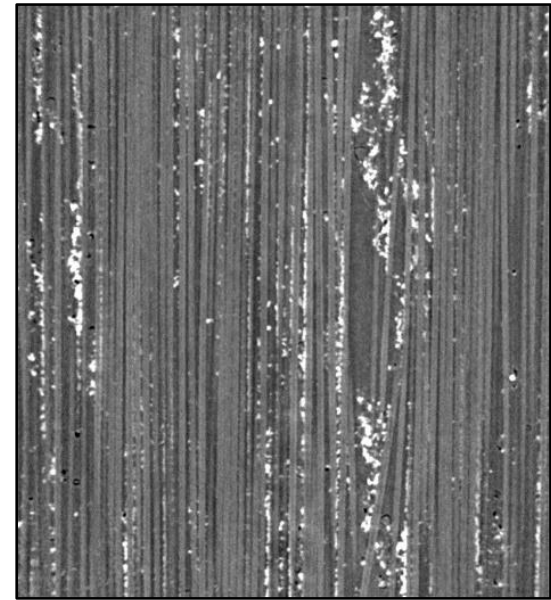

(b) Raw CT slice 130/230

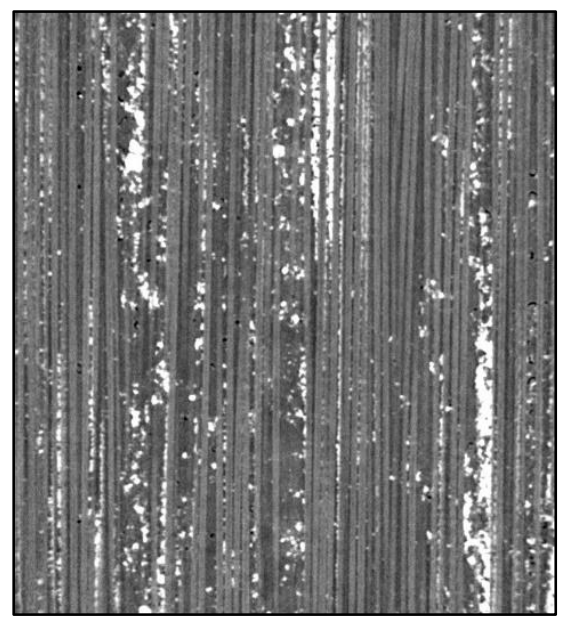

(d) Raw CT slice 179/230

Figure 22 - Raw CT data slices showcasing changes in material microstructure, potentially accountable for an uneven strain distribution at fibre-level. Each slice has a FOV of $795 \times 885$ voxels ( 0.64 $\mu$ m voxel size): (a)

illustrating tow-level misalignment, (b) large resin-rich channel coupled with fibre direction errors, (c) multiple, parallel resin-rich channels and fibre misalignment, (d) large resin-rich channel and fibre misalignment. All slices show a nonuniform particle distribution.

\section{Summary and conclusions}

A comprehensive DVC study was performed at sub-micrometre resolution $(\sim 0.64 \mu \mathrm{m})$ on UD carbon-fibre composite plies, to validate the methodology by comparison to the analytical result for the through-thickness strain distribution in a state of pure bending. To counteract the sub-optimal microstructures found in conventional unidirectional CFRPs for the application of DVC, particularly along the fibre direction, $\mathrm{BaTiO}_{3}$ particles with a mean size of $400 \mathrm{~nm}$ and volume fraction of $\sim 1.44 \%$ were introduced in the matrix. Micro-focus CT measurements were acquired from a simple beam-type specimen subjected to a four-point flexural test, which led to a well-defined strain distribution. A rigid body displacement test was also performed to determine the experimental error. The test also confirmed that that specimen translation parallel to the fibre direction is in line with the applied displacements, obviating a key problem in applying DVC to such uniformly aligned microstructures. DVC results were 
compared against the classical beam theory in incrementally decreasing averaging schemes, and at various spatial resolutions: 120 voxels, 100 voxels and 80 voxels sub-set size, with $50 \%$ overlap applied between adjacent sub-sets. Good agreement was found between the Euler-Bernoulli beam theory and DVC output providing that the mean of three or more standalone sub-sets was taken, demonstrating the effectiveness of DVC in measuring local strains parallel to the fibre direction in UD laminae. Edgeartefacts were shown to introduce subtle nonlinearities in the measurements, illustrating that any DVC results located at the edge of the cropped/masked volume image must be treated with caution. Deviations from the classical beam theory, exceeding that of the experimental error, were observed when sampling was performed with single 120 voxel size sub-sets. This represents a practical lower limit for the spatial resolution using the technique employed in the current work, and may also be attributed to variations in the material microstructure. The methodology provides a framework for accessing unprecedented opportunities to identify the local strain distributions before and after damage formation in composite materials, in 3D, at relatively high spatial resolutions. Future work will report the performance of the fiducial-adapted material and mechanistically consistent measurements in relation to local fibre failure events, such as strain transfer lengths. To eliminate some of the present caveats, in particular related to spatial and strain resolutions achievable, detailed analyses will be conducted on SRCT data. This provides the opportunity to refine the understanding of composite tensile failure processes alongside the various other forms of composite damage that exist, and is in turn expected to provide a valuable tool for developing and validating micromechanics models for composite damage and failure.

\section{Acknowledgements}

The research leading to these results has been conducted within the framework of the FiBreMoD project and has received funding from the European Union's Horizon 2020 research and innovation programme under the Marie Skłodowska-Curie grant agreement No. 722626.

The authors acknowledge contributions from institutions and staff: LaVision $\mathrm{GmbH}$ for the off-site software supply and technical support from Dr. David Hollis, Dr. Alex Nila and Dr. Manuel Grewer. Material manufacturing has received significant scientific contribution from the Composite Materials Group (CMG) at KU Leuven: Dr. Yentl Swolfs, Mr. Christian Breite, Mr. Arsen Melnikov and Mr. Yoran Geboes. Technical support from the mechanical workshop and composite laboratory staff from the Department of Materials Engineering at KU Leuven is also recognized. Accompanying acknowledgement is given to Dr. Richard Boardman, Dr. Kathryn Rankin, Dr. Orestis Katsamenis and Mr. Nicholas Hale from the $\mu$-VIS X-Ray Imaging Centre at the University of Southampton. Dr. Daniel Bull from the Engineering Materials Group at the University of Southampton is credited with trial CT scanning assistance. Credit is further extended to Mr. Simon Beever - University of Southampton Engineering Design and Manufacturing Centre (EDMC) and Mr. Adrian Walker - Safire Associates UK for the short notice water-jet cutting of various components required for the experimental work. 


\section{References}

[1] United Nations, "Paris Agreement," 2015.

[2] K. Van Acker, I. Verpoest, J. De Moor, J.-R. Duflou and W. Dewulf, "Lightweight materials for the automotive: environmental impact analysis of the use of composites," in The 3rd International Seminar on Society \& Materials - SAM3, Freiberg, Germany, 2009.

[3] A. J. Moffat, P. Wright, J.-Y. Buffiere, I. Sinclair and S. M. Spearing, "Micromechanisms of damage in $0^{\circ}$ splits in a [90/0]s composite material using synchrotron radiation computed tomography," Scripta Materialia, vol. 59, no. 10, pp. 1043-1046, 2008.

[4] P. Wright, A. J. Moffat, I. Sinclair and S. M. Spearing, "High resolution tomographic imaging and modelling of notch tip damage in a laminated composite," Composites Science and Technology, vol. 70, no. 10, pp. 1444-1452, 2010.

[5] A. E. Scott, M. N. Mavrogordato, P. Wright, I. Sinclair and S. M. Spearing, "In situ fibre fracture measurement in carbon-epoxy laminates using high resolution computed tomography," Composites Science and Technology, vol. 71, no. 12, pp. 1471-1477, 2011.

[6] S. C. Garcea, I. Sinclair, S. M. Spearing and P. J. Withers, "Mapping fibre failure in situ in carbon fibre reinforced polymers by fast synchrotron X-ray computed tomography," Composites Science and Technology, vol. 149, pp. 81-89, 2017.

[7] S. Rosini, M. N. Mavrogordato, O. Egorova, E. S. Matthews, S. E. Jackson, S. M. Spearing and I. Sinclair, "In situ statistical measurement of local morphology in carbon-epoxy composites using synchrotron X-ray computed tomography," Composites Part A, vol. 125, pp. 1-14, 2019.

[8] B. Pan, K. Qian, H. Xie and A. Asundi, "Two-dimensional digital image correlation for in-plane displacement and strain measurement: a review," Measurement Science and Technology, vol. 20, no. 6, pp. 1-17, 2009.

[9] B. K. Bay, T. S. Smith, D. P. Fyhrie and M. Saad, "Digital volume correlation: Three-dimensional strain mapping using X-ray tomography," Experimental Mechanics, vol. 39, no. 3, pp. 217-226, 1999.

[10] J. Adam, M. Klinkmüller, G. Schreurs and B. Wieneke, "Quantitative 3D strain analysis in analogue experiments simulating tectonic deformation: Integration of X-ray computed tomography and digital volume correlation techniques," Journal of Structural Geology, vol. 55, pp. 127-149, 2013.

[11] F. Xu, "Quantitative characterization of deformation and damage process by digital volume correlation: A review.," Theoretical \& Applied Mechanics Letters, vol. 8, no. 2, pp. 83-96, 2018.

[12] E. Schöberl, M. N. Mavrogordato, I. Sinclair and S. M. Spearing, "The mapping of damage behaviour in a unidirectional composite subjected to uniaxial loading using digital volume correlation," in The 12th International Conference on Composite Science and Technology, Sorrento, Italy, 2019. 
[13] E. Schöberl, C. Breite, A. Melnikov, Y. Swolfs, M. N. Mavrogordato, I. Sinclair and S. M. Spearing, "Mapping strains and fibre fracture in carbon fibre composites using in situ digital volume correlation," in The 22nd International Conference on Composites Materials, Melbourne, Australia, 2019.

[14] P. Lecomte-Grosbras, J. Réthoré, N. Limodin, J.-F. Witz and M. Brieu, “Three-dimensional investigation of free-edge effects in laminate composites using X-ray tomography and digital volume correlation," Experimental Mechanics, vol. 55, no. 1, pp. 301-311, 2015.

[15] G. Borstnar, F. Gillard, M. N. Mavrogordato, I. Sinclair and S. M. Spearing, "Three-dimensional deformation mapping of Mode I interlaminar crack extension in particle-toughened interlayers," Acta Materialia, vol. 103, pp. 63-70, 2015.

[16] R. Brault, A. Germaneau, J. C. Dupré, P. Doumalin, S. Mistou and M. Fazzini, "In-situ analysis of laminated composite materials by X-ray micro-computed tomography and digital volume correlation," Experimental Mechanics, vol. 53, pp. 1143-1151, 2013.

[17] M. Palanca, L. Cristofolini, E. Dall'Ara, M. Curto, F. Innocente, V. Danesi and G. Tozzi, “Digital volume correlation can be used to estimate local strains in natural and augmented vertebrae: An organ-level study," Journal of Biomechanics, vol. 49, no. 16, pp. 3882-3890, 2016.

[18] F. Gillard, R. P. Boardman, M. N. Mavrogordato, D. Hollis, I. Sinclair, F. Pierron and M. Browne, "The application of Digital Volume Correlation (DVC) to study the microstructural behaviour of trabecular bone during compression," Journal of the Mechanical Behavior of Biomedical Materials, vol. 29, pp. 480-499, 2014.

[19] L. Liu and E. F. Morgan, "Accuracy and precision of digital volume correlation in quantifying displacements and strains in trabecular bone," Journal of Biomechanics, vol. 40, no. 15, pp. 3516-3520, 2007.

[20] F. Pierron, S. A. McDonald, D. Hollis, J. Fu, P. J. Withers and A. Alderson, "Comparison of the mechanical behaviour of standard and auxetic foams by X-ray computed tomography and digital volume correlation," Strain, vol. 49, no. 6, pp. 467-482, 2013.

[21] S. P. Timoshenko, Strength of materials. Part 1 - Elementary theory and problems, 2nd ed., New York City, NY, USA: David Van Nostrand Company Inc., 1940, pp. 88-97.

[22] Toray Industries Inc., Toray Composite Materials, Tokyo, Japan, 2019.

[23] US Research Nanomaterials Inc., Micron Powders, Houston, TX, USA, 2019.

[24] Sicomin, Epoxy Systems, Châteauneuf-les-Martigues, France, 2018.

[25] Ultrawave Ltd., U100/U100H Ultrasonic Cleaning Bath, Cardiff, UK, 2017.

[26] ASTM International, "Standard Test Method for Flexural Properties of Polymer Matrix Composite Materials D 7264/D 7264M - 07," West Conshohocken, PA, USA, 2007.

[27] Carl Zeiss AG, Xradia 510 Versa, Oberkochen, Germany, 2018. 
[28] LaVision GmbH, DaVis v10 StrainMaster (Digital Volume Correlation), Göttingen, Germany, 2018.

[29] M. P. Fernández, A. H. Barber, G. W. Blunn and G. Tozzi, “Optimization of digital volume correlation computation in SR-microCT images of trabecular bone and bone-biomaterial systems," Journal of Microscopy, vol. 272, no. 3, pp. 213-228, 2018.

[30] K. Madi, G. Tozzi, Q. H. Zhang, J. Tong, A. Cossey, A. Au, D. Hollis and F. Hild, "Computation of full-field displacements in a scaffold implant using digital volume correlation and finite element analysis," Medical Engineering \& Physics, vol. 35, no. 9, pp. 1298-1312, 2013.

[31] E. M. C. Jones and M. A. Iadicola, "A good practices guide for digital image correlation," International Digital Image Correlation Society (iDICs), 2018.

[32] M. Palanca, G. Tozzi, L. Cristofolini, M. Viceconti and E. Dall'Ara, "Three-dimensional local measurements of bone strain and displacement: comparison of three digital volume correlation approaches," Journal of Biomedical Engineering, vol. 137, no. 7, pp. 1-14, 2015.

[33] O. Jiroušek, I. Jandejsek and D. Vavřík, "Evaluation of strain field in microstructures using micro-CT and digital volume correlation," in The 12th International Workshop on Radiation Imaging Detectors, Cambridge, UK, 2011.

[34] A. Buljac, T. Taillandier-Thomas, L. Helfen, T. F. Morgeneyer and F. Hild, "Evaluation of measurement uncertainties of digital volume correlation applied to laminography data," The Journal of Strain Analysis, vol. 53, no. 2, pp. 49-65, 2018.

[35] GOM GmbH, GOM Inspect 2018, Braunschweig, Germany, 2018.

[36] J. Schindelin, I. Arganda-Carreras, E. Frise, V. Kaynig, M. Longair, T. Pietzsch, S. Preibisch, C. Rueden, S. Saalfeld, B. Schmid, J.-Y. Tinevez, D. J. White, V. Hartenstein, K. Eliceiri, P. Tomancak and A. Cardona, "Fiji: an open-source platform for biological-image analysis," Nature Methods, vol. 9, pp. 676-682, 2012.

[37] Volume Graphics GmbH, VGStudio Max 2.5, Heidelberg, Germany.

[38] Dassault Systèmes SE, SOLIDWORKS, Vélizy-Villacoublay, France, 2015.

[39] S. Yaofeng and J. H. L. Pang, "Study of optimal subset size in digital imagecorrelation of speckle pattern images," Optics and Lasers in Engineering, vol. 45, no. 9, pp. 967-974, 2007.

[40] Tampere University of Applied Sciences (TAMK), "Digital Image Correlation - IMPACT Multiscale Materials Testing Research Group," [Online]. Available: https://research.tuni.fi/impact/equipment/digital-image-correlation/. [Accessed 3001 2020]. 